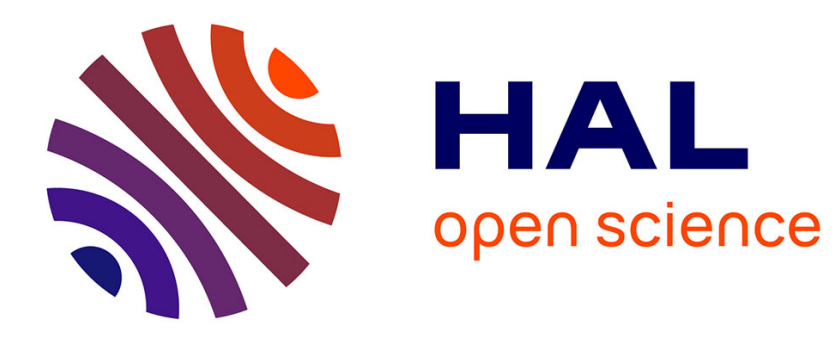

\title{
An Expression of Classical Dynamics
}

Jean Jacques Moreau

\section{To cite this version:}

Jean Jacques Moreau. An Expression of Classical Dynamics. Annales de l'Institut Henri Poincaré C, Analyse non linéaire, 1989, 6, pp.1 - 48. 10.1016/S0294-1449(17)30022-7 . hal-01789036

\section{HAL Id: hal-01789036 https://hal.science/hal-01789036}

Submitted on 9 May 2018

HAL is a multi-disciplinary open access archive for the deposit and dissemination of scientific research documents, whether they are published or not. The documents may come from teaching and research institutions in France or abroad, or from public or private research centers.
L'archive ouverte pluridisciplinaire HAL, est destinée au dépôt et à la diffusion de documents scientifiques de niveau recherche, publiés ou non, émanant des établissements d'enseignement et de recherche français ou étrangers, des laboratoires publics ou privés. 


\title{
AN EXPRESSION OF CLASSICAL DYNAMICS
}

\author{
J. J. MOREAU \\ Laboratoire de Mécanique Générale des Milieux Continus, \\ Unité Associée au C.N.R.S. 1214 , \\ Université des Sciences et Techniques du Languedoc, \\ MONTPELLIER, France.
}

\begin{abstract}
The proposed formulation extends the Euler variable approach, classical in Continuum Mechanics, up to make it valid for such singular systems as, for instance, a single mass-point. The key concept is the kinetic tensor measure of the investigated material, relative to some window in time-space. This is first developed in the framework of Galilean time-space. In that case, the fundamental equation involves the four-dimensional vector distribution divergence of the kinetic tensor measure. It is shown, in particular, how the initial conditions of an evolution problem or the conf inement of the investigated system by a given boundary, possibly with shocks, may be described through adequate terms in the fundamental equation. In order to develop similar procedures in the Riemannian manifold setting of Analytical Dynamics, one introduces the differential operator equilibrium, acting on the doubly contravariant symmetric tensor measures of the manifold. This operator receives a variational interpretation, in terms of the transport by test flows. Thereby, the connection of the proposed formulation of Dynamics with Hamilton's principle is explained.
\end{abstract}




\section{Introduction}

Studies devoted to the existence or the regularity of solutions to problems arising from Physics, in particular from Mechanics, commonly begin with stating what actually will be meant by a solution. Except in some theoretical chapters of Physics, the generally accepted conceptual background does not generate mathematical problems in a form allowing for the application of the current methods of Functional Analysis to the investigation of solutions. This is the price one has to pay for conducting physical inspection in a language which involves only some familiar mathematical concepts, such as $C^{1}$ functions, functions with jumps on smooth surfaces, etc...

In our views, this state of affairs cannot be expected to change rapidly. Bridging the gap between Functional Analysis and the principles on which the respective chapters of Physics are founded would surely need a lot of technicalities, with dissuasive effect on the majority of the public. And meanwhile, the progresses of Functional Analysis would be liable to reveal alternative approaches, with more promising prospect.

Generally, axiomatic improvement looks like the ever unfinished job of cleaning after the action. As long as a scientific domain is alive, the investigation of facts in its active fringe is never entirely conducted through the logical application of previously stated "principles", but involves inductive thinking. Only afterwards are the principles adjusted, so as to permit the deductive arrangement of the findings. This is true, even in such a domain as Mechanics, the theoretization of which has begun early. The treatment of adhesion [3] or that of continuous media with microstructure (see, e.g. [1]), among other current examples, illustrate this observation.

No attempt is made in this paper at systematically constructing an updated axiomatic of Classical Mechanics. This would be a specialist's work 
and a heavy task, for the number of axioms needed to make a complete system is greater than it seems at first glance. There only will be displayed certain chains of mathematical properties which, some day, could play a significant role in such a construction. In the meantime, the formulation we propose is more modestly expected to reduce the distance between the statement of some mechanical problems and their mathematical study.

The elementary example of a continuous medium with $C^{1}$ velocity field is used in Sec. 2 to introduce the main concepts. It is shown how the balance of mass and the balance of momentum of the investigated material may be condensed into a four-dimensional equation. This introduces a doubly contravariant symmetric tensor measure, called the kinetic tensor measure, relative to a chosen part of time-space called a window. The latter is the geometric container of the mechanical information that one intends to treat. It does not necessarily involve the same material particles at every instant. The fundamental equation, which is asserted to govern Dynamics, puts forward the divergence of the kinetic tensor measure, a differential operator understood in the sense of Schwartz's Distributions.

Galliean invariance is an essential feature of Classical Mechanics. In order to make sure that the proposed formulation meets this requirement, we choose, in Sec. 3, to expose it in the coordinate-free setting of the Galilean time-space $\mathbb{G}$.

Sec. 4 explains how this formalism applies, in particular, to the dynamics of a single particle. The time-dependent efforts acting on it are represented by a vector distribution of order $\leqslant 1$ on $\mathbb{G}$. In the most significant cases, this order actually equals zero, i.e. the said distribution is a four-dimensional (Radon) measure. Then it is found that the velocity vector of the particle is a function of time with locally bounded variation and the motion is governed by a measure differential equation [16][18]. This includes as a special case the traditional treatment of shocks through the concept of percussion. 
It is also the example of a single particle which is used, in Sec. 5 , to demonstrate how the choice of an adequate window allows one to describe the initial conditions of an evolution problem by means of terms in the fundamental equation. Depending on the decision made, of including or not the initial instant $t_{0}$ in the investigation, it is either the limit of the velocity vector on the left or on the right of $t_{0}$ which has to be considered as "initial velocity".

In Sec. 6 is formalized the confinement of the particle by a boundary, a typical example of unilateral constraint. Here again, the concept of window proves essential.

One of the reasons for having focused attention on the case of a single particle is that the traditional Analytical Dynamics formally reduces arbitrary systems to moving points in Riemannian manifolds. The extension of the preceding formalism to this case calls for discussing tensor measures or tensor distributions in such a manifold and for comparing different generalizations of the divergence operator, which, in the Galilean setting, used to play the central part. This is the object of Sec. 7, where the equiliorium operator is introduced. By definition, the latter equals the negative transpose of the deformation, a standard differential operator in Continuum Mechanics.

This is used in Sec. 8 , where a mechanical system of finite freedom is considered, with a Riemannian manifold $Q$ as its set of possible configurations. It is found that, by applying, in the product manifold $\mathbb{R} \times \mathcal{Q}$, the equilibrium operator to the corresponding kinetic tensor measure, and equalling the result to the covector measure on $\mathbb{R} \times \mathbb{Q}$ which represents the efforts acting on the system, one recovers the Lagrange equations. An advantage of this procedure is that, similarly to what has been observed in Sec. 4, it keeps meaningful in some significant nonsmooth motions, then leading to measure differential equations. Such is the case for motions involving collisions with a boundary. Thereby, the setting previously adopted by the author in developing numerical methods for the dynamics of 
systems presenting unilateral contacts (possibly with dry friction) [17] receives a theoretical foundation.

The rest of the paper is aimed at connecting the proposed formulation of Dynamics with the variational principle of Hamilton. This is achieved through the transport method, formerly used by the author on various purposes $[10][13][15]$. In this method, variations are imparted to the investigated objects by having them carried along a certain class of flows. In particular, the equilibrium operator, acting on the doubly contravariant tensor measures of the concerned manifold, receives in that way a variational meaning. The advantage of such a procedure over the traditional calculus of variations is that it requires less smoothness of the investigated objects. The form this gives to Hamilton's principle is still valid for motions with non differentiable velocity function. The latter is only assumed to have locally bounded variation, allowing, in particular for the presence of shocks.

\section{A heuristic example.}

Let us first consider a continuous medium whose motion, relative to some orthonormal inertial axes $0 x^{1} x^{2} x^{3}$, is smooth enough for the three components $u^{i}$ of the velocity field and the density $\rho$ to be $c^{1}$ functions of the time variable and of the $x^{i}$ coordinates. Let us denote by $x^{0}$ the time variable and agree, for all the sequel, that Greek indices will take their values in $\{0,1,2,3\}$, while Latin ones will take theirs in $\{1,2,3\}$.

It is known that the three equations of momentum balance and the equation of mass conservation may be combined, so as to be condensed into the equivalent four-dimensional writing

$$
\left(\rho u^{\alpha} u^{\beta}\right)_{\beta}=f^{\alpha} \text {. }
$$

Here, ${ }_{\beta}$ denotes the partial derivation with respect to $x^{\beta}$ and, by convention, $u^{0} \equiv 1$. For $\alpha>0$, the expression $f^{\alpha}$ represents the component of rank $\alpha$ of the three-dimensional volume density of efrort. In common cases, this vector field equals the volume density of external effort, plus the divergence of 
the Cauchy stress tensor field. Besides, $f^{0} \equiv 0$, unless a supply or extraneous material is imagined, at the rate of $f^{0}$ unit of mass per unit of timexvolume (in that case, the velocity of the supplied material must also be given, inducing a contribution in $\left.f^{1}, f^{2}, f^{3}\right)$.

Let us denote by $x$ the Euclidean linear space where $x^{1}, x^{2}, x^{3}$ are orthonormal coordinates; then $x^{0}, x^{1}, x^{2}, x^{3}$ may be seen as orthonormal coordinates in the product space $\mathbb{R} \times X$, itself equipped in the standard way with a Euclidean metric. One may interpret the left-hand side of (2.1) as expressing the four components, indexed by $\alpha$, of the vector field divergence of the tensor field in $\mathbb{R} \times x$ with components $\rho u^{\alpha} u^{\beta}$.

If the motion is not smooth enough for the partial derivatives to exist in the elementary sense, there is no doubt that the dynamics of the considered material is correctly expressed by understanding these derivatives "in the sense of Schwartz's Distributions in $\mathbb{R} \times X$ ". This actually is an abuse of language, since never a function equals a distribution. What in fact constitutes a distribution in the sense of L. Schwartz, is the measure possessing the considered function as density, relative to Lebesgue's measure. of course, the function has to be locally integrable with respect to the latter.

As an example, in a situation familiar to Fluid Mechanists, one may check that the Distribution formalism, applied to (2.1), readily yields the balance equations of mass and momentum across a shock wave in an inviscid fluid [10].

Generally, we propose to formulate in the following way the dynamics of some matter present in a subset $W$ of the timexspace $\mathbb{R} \times X$. The notation $W$ here is chosen as a reminder of window, a denomination we shall later explain in more detail.

First, a nonnegative real measure in $\mathbb{R} \times X$, concentrated on $W$, called the presence measure of the said matter, has to be defined. Let us denote it by $\theta$. The formulation in view makes sense provided the velocity components $u^{\alpha}$ (with $u^{0} \equiv 1$, by convention) are elements of $L_{\text {loc }}^{2}(\mathbb{R} \times X, \theta ; \mathbb{R})$. 
We shall admit that, as rar as motions of this sort are concerned. the dunamics or the considered matter is governed by

$$
\left(u^{\alpha} u^{\beta} \theta\right)_{, \beta}=F^{\alpha} \text {. }
$$

On the left-hand side are the components of the four-dimensional vector distribution divergence of the symmetric tensor measure $\mathrm{C}$, with components $C^{\alpha \beta}=u^{\alpha} u^{\beta} \theta$. In other words, $C$ possesses as density, relative to $\theta$, the tensor function $u \otimes u \in L_{l o c}^{1}\left(\mathbb{R} \times X, \theta ;(\mathbb{R} \times X) \otimes_{s}(\mathbb{R} \times X)\right)$. We propose to call $C$ the kinetic measure of the considered matter.

For this equality of distributions to hold, the right-hand members $\mathrm{F}^{\alpha}$, $\alpha \in\{0,1,2,3\}$, have to be elements of $\mathcal{D}^{\prime 1}(\mathbb{R} \times X, \mathbb{R})$, i.e. to be the components of a vector distribution $F$ on $\mathbb{R} \times X$, with order $\leqslant 1$. This vector distribution conveys all the information needed about the internal and external efforts that the considered matter experiences and about the possible loss or collection of material (for instance through the boundary of $w$ : we shall come back to this in Secs. 6 and 7). Actually, the special case where $F$ happens to be a distribution of order zero, i.e. a (Radon) measure, will prove the most significant.

REMARK 2.1. In our introductory example, the presence measure admitted the real function $\varrho$ as density, relative to the Lebesgue measure of $\mathbb{R} \times X$. It is clear from this example that the approach of Dynamics we are developing extends what, in Cont inuum Mechanics, is commonly called the treatment of a problem in Euler variables. In such a treatment, the description of motion is primarily done through the velocity vector of the matter, at every point of the concerned region of timexspace. Whether the vector field with components $\left(1, u^{1}, u^{2}, u^{3}\right)$ possesses integral lines in $\mathbb{R} \times x$, defining the motion of individual particles, becomes a secondary question. This is a realistic attitude, since the velocity vector is nothing but an average value, referring to the underlying agitation of microscopic objects. In reality, continuous media (principally those which are qualified as fluids) evolve with a certain amount of intradiffusion, so the individuation of particles can only 
emerge as an approximate concept. The Euler variable treatment proves also well adapted to calculating the average flow of a microscopically heterogeneous fluid (e.g. a flow with suspended small objects or involving multiphasic micro-structure). In contrast, most models of deformable solids require the individuation of particles.

Anyway, it is rather unexpected to use the Euler variable approach in formulating also the dynamics of a single mass-point, as we shall do in Sec. 4 .

REMARK 2.2 The Dynamics of mixtures suggests to generalize the preceding formulation, up to accept as $C$ a symmetric tensor measure which no more equals a "tensor square". We mean that, when $C$ is represented in the form $C_{\mu}^{\prime} \mu$, where $\mu$ is a nonnegative real measure and

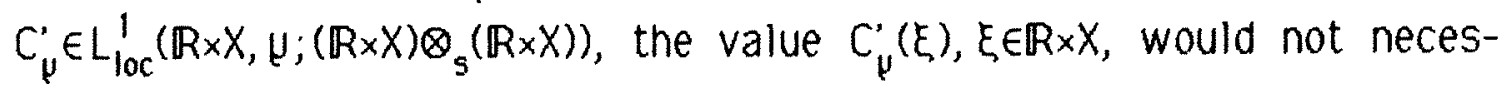
sarily have, for $y$-almost every $\xi$, the form $v(\xi) \otimes v(\xi)$, with $\vee$ denoting a vector field. Similar remark applies to Stochastic Dynamics : in order to take into account the data uncertainty, one may be led to treat, instead of a single motion, some probabilized collection of them. Then $C$ is replaced by some probabilized average of the corresponding tensor measures; this in general is not a tensor square. Another source of interest of this collective approach to dynamical problems could be to disregard some singular solutions, by considering them as "non generic".

Even so, it seems to us that the nonnegativity of the quadratic form with matrix $C_{\mu}^{\alpha \beta}(\xi)$, which trivially holds in the foregoing, has to be placed among the principles of Dynamics. An argument in favor of this postulate may be found in [15], an introduction to the general use of the Transport Method. In this method, whose application to the present situation is described in Sec.10 below, the possibly nonsmooth solutions to some field equations are characterized as yielding zero variation rate for a certain functional in a certain type of alteration processes. The above nonnegativity then arises from the study of the second variation rate and, roughly speaking, may be interpreted as a "stability" requirement. 
The nonnegativity postulate also has the merit of protecting one from the temptation of accepting as $C$ some tensor distribution with order $h>0$. In fact, if $C^{\alpha \beta} \in \mathcal{D}^{\prime h}(\mathbb{R} \times X, \mathbb{R})$, with $C^{\alpha \beta}=C^{\beta \alpha}$, are the components of a tensor distribution, the nonnegativity assertion becomes $\left\langle C^{\alpha \beta}, \varphi_{\alpha} \varphi_{\beta}\right\rangle \geqslant 0$, holding for every $\varphi \in \mathcal{D}^{h}(\mathbb{R} \times X, \mathbb{R} \times X)$. This property may be shown [15] to require $h=0$.

REMARK 2.3 The definition of a Euclidean metric in $\mathbb{R} \times X$, the time-space, rests on the choice of time and length units. $A$ unit-free construction would be possible, at the price of more complicated notations. We shall in the sequel go on assuming that the physical units are fixed.

\section{The Galilean setting.}

Galilean invariance is a dominant feature of Classical Dynamics. Basically, it consists in saying that, given some inertial reference frame (i.e. a frame in which the familiar momentum equation holds; this is also called a Galilean frame), any other frame whose motion, relative to it, is a rectilinear and uniform translation is inertial too. The underlying trivial fact is that, if a moving point possesses an acceleration with regard to the former frame, then the same vector is also the acceleration of this point with regard to the latter.

Rather than asking for "invariance" under some class of operations, we shall in this paper adopt the synthetic approach. This consists in describing first some geometrical structure, providing the framework in which all subsequent assertions are to be formulated. Then, automatically, these assertions will be "invariant under the automorphism group of the considered structure". In other words, instead of checking that a statement is "frame-indifferent", we prefer to exhibit a formulation of it in a "frame-free" language.

Considering an affine space $\mathbb{G}$, we shall denote by $\mathbb{G}^{\circ}$ the linear space of the corresponding vectors (this is consistent with the notations used, about manifolds, in further sections: $\mathbb{G}^{\prime}$ in fact equals the tangent space to $\mathbb{G}$ at any point). For a differentiable function $f: \mathbb{G} \rightarrow \mathbb{R}$, the gradient $\nabla f$, i.e. 
the tangent linear map $\mathbb{G} \rightarrow \mathbb{R}$, at a point is an element of the dual $\mathbb{G}^{\circ *}$ of $\mathbb{G}^{\prime}$ The duality bilinear form will be denoted by a dot and the corresponding orthogonality by ${ }^{\perp}$.

DEFINITION 3.1. A Galilean time-space (or Galilean event space) is a four-dimensional arfine space, say $\mathbb{G}$, specialized by the fixation of the following objects:

$1^{\circ}$ A non-constant affine function $\mathbb{D}: \mathbb{G} \rightarrow \mathbb{R}$, called date.

$2^{\circ}$ A Euclidean metric in the subspace $\mathbb{E}=(\nabla \mathbb{D})^{+}$of $\mathbb{G}^{\prime}$.

The elements of $\mathbb{E}$ are called spatial vectors.

The trick of using the Euclidean structure of $\mathbb{E}$, in order to identify this linear space with its dual $\mathbb{E}^{*}$, will not be applied for the moment.

DEFINITION 3.2. A moving point is a mapping. say $\pi$, of a real interval $T$ ( called an interval of time) to $\mathbb{G}$, verifying

$$
\forall \mathrm{t} \in \mathrm{T}: \mathbb{D}(\pi(\mathrm{t}))=\mathrm{t} \text {. }
$$

If $\pi$ is differentiable at some $\mathrm{t}$, the derivative $\mathbf{u}=\dot{\pi}(\mathrm{t})$, an element of $\mathbb{G}^{\prime}$, is called the absolute velocity of the moving point at instant $\mathrm{t}$.

From (3.1), it results that

$$
\text { u. } \nabla \mathbb{D}=1 \text {. }
$$

If $\pi$ is an arfine mapping, so that $\mathrm{u}$ is constant with regard to $\mathrm{t}$, the moving point is said to have an inertial motion.

DEFINITION 3.3. A Cartesian coordinate system of $\mathbb{G}$, say $0 x^{0} x^{1} x^{2} x^{3}$, is called Galilean or inertial if

$$
\mathbb{D}(0)=0
$$

and if its base vectors $\mathbf{i}_{(0)}, \mathbf{i}_{(1)}, \mathbf{i}_{(2)}, \mathbf{i}_{(3)} \in \mathbb{G}^{\prime}$ satisfy

$$
\mathbf{I}_{(0)}, \nabla \mathbb{D}=1 \quad \text { and } \quad \mathbf{I}_{(1)}, \nabla \mathbb{D}=\mathbf{I}_{(2)}, \nabla \mathbb{D}=\mathbf{i}_{(3)}, \nabla \mathbb{D}=0
$$

This, in particular, implies that $\mathbf{i}_{(1)}, \mathbf{i}_{(2)}, \mathbf{i}_{(3)}$ belong to $\mathbb{E}$. One says that the axes $0 x^{1}, O x^{2}, O x^{3}$ have spatialdirections.

Instead of conditions (3.4), one may equivalently introduce the base of 
$\mathbb{G}^{\prime *}$ adjoint to $\left\{\mathfrak{i}_{(0)}, \mathfrak{i}_{(1)}, \mathfrak{i}_{(2)}, \mathbf{i}_{(3)}\right\}$ and assert that, relatively to this base, the covector $\nabla \mathbb{D}$ has components $1,0,0,0$.

Under these conditions, for any point $\xi$ of $\mathbb{G}$ with coordinates $x^{0}, x^{1}, x^{2}, x^{3}$, one has $\mathbb{D}(\xi)=x^{0}$.

As soon as a coordinate system of this sort has been chosen, every moving point may be described by giving the four coordinates $\pi^{\alpha}(t)$ of $\pi(t)$, with $\pi^{0}(t) \equiv t$. If, in particular, the functions $\pi^{1}, \pi^{2}, \pi^{3}$ are constant, the moving point is said rixed in the spatial frame $0 x^{1} x^{2} x^{3}$, or to be a particle attached to this frame. Then the motion of this point is inertial, with absolute velocity equal to $\mathbf{i}_{(0)}$. Such is the aspect that the concept of an inertial reference irame takes on in the present formalization. Practically, $x^{1}, x^{2}, x^{3}$ are interpreted as Cartesian coordinates in some three-dimensional affine space $x$, whose points are identified with the particles attached to the frame. One says that $X$ is an inertial reference space.

Observe that the Euclidean metric of $\mathbb{E}$ and the possible orthonormality of $\mathbf{i}_{(1)}, \mathbf{i}_{(2)}, \mathbf{i}_{(3)}$ with regard to it play no part in what precedes. They will only become significant in Remarks 3.6 and 3.7 below.

When a reference space $X$ has been specified as above, every $\xi \in \mathbb{G}$ lets itself univocally be represented in the form $\left(x^{0}, x\right)$, with $x^{0}=\mathbb{D}(\xi) \in \mathbb{R}$ and $x \in X$. For a moving point $\pi$, it proves expedient to use the writing

$$
\pi(t) \simeq(t, p(t)) \text {, with } p(t) \in X
$$

If $\mathbf{u}=\dot{\pi}(t)$ exists, the difference $u_{x}=\mathbf{u}-\mathbf{i}_{(0)}$ belongs to $\mathbb{E}$. By definition, this is the velocity of the moving point relative to the reference space $x$. Clearly, the space of the vectors of $x$ may be identified with $\mathbb{E}$, so this relative velocity is found equal to the derivative $\dot{p}(t)$.

We now are going to show that the formulation of Classical Dynamics proposed in Sec.2 makes sense in the setting of Galllean time-space.

A window is a subset $\mathbb{W}$ of $\mathbb{G}$. With the matter present in it, the kinetic tensor measure is associated. Its construction starts with the defi- 
nition of the presence measure of the said matter, a nonnegative real measure, say $\theta$, concentrated on $W$. Afterwards, the absolute velocity field is introduced as an element, say $\mathbf{u}$, of $L_{\text {loc }}^{2}\left(\mathbb{G}, \theta ; \mathbb{G}^{\prime}\right)$, satisfying $(3.2)$ $\theta$-a.e.. Then, the kinetic tensor measure is, by definition, the element $C=\mathbf{u} \otimes \mathbf{u} \theta$ of $\mathcal{D}^{\circ}\left(\mathbb{G}, \mathbb{G}^{\prime} \otimes_{s} \mathbb{G}^{\prime}\right)$.

The classical discussion of covariance and contravariance, when partial derivation with respect to Cartesian coordinates in an arbitrary affine space is involved, entails that the distributions $C^{\alpha \beta},{ }_{\beta} \in \mathcal{D}^{\prime}(\mathbb{G}, \mathbb{R})$ equal the components of an element of $\mathcal{D}^{\prime 1}\left(\mathbb{G}, \mathbb{G}^{\prime}\right)$, i.e. a (contravariant) vector distribution of $\mathbb{G}$, independent of the Cartesian coordinate system in use. This is the divergence of $\mathrm{C}$, in the sense of the natural connection of the affine space $\mathbb{G}$. This distribution may also be constructed without reference to any coordinate frame, as the functional assigning to every $\varphi \in \mathcal{D}^{\prime}(\mathbb{G}, \mathbb{R})$ an element of $\mathbb{G}^{\prime}$ as follows:

$$
\varphi \rightarrow-\langle T, \nabla \varphi\rangle=-\int \mathbf{u}(\mathbf{u}, \nabla \varphi) d \theta \text {. }
$$

Then. the formulation proposed in Sec. now may be translated into the frame-free writing

$$
\operatorname{div} C=F .
$$

It must be kept in mind that, in spite of the investigation being restricted to the subset $W$, distributions here and the differential operator div are understood in the sense of the whole space $\mathbb{G}$.

The distribution $F \in \mathcal{D}^{\prime 1}\left(\mathbb{G}, \mathbb{G}^{\prime}\right)$ on the right-hand side will, in practice, equal a sum of terms conveying various pieces of information about the physical effects that the matter investigated in the window $W$ experiences. The following remarks play a significant role in discussing these terms.

The contracted multiplication of $C$ by the constant covector field $\nabla D$ yields a vector measure on $\mathbb{G}$, concentrated in $W$,

$$
\text { C. } \nabla \mathbb{D}=(\nabla \mathbb{D} \cdot \mathbf{u}) \mathbf{u} \theta=\mathbf{u} \theta \text {. }
$$

The divergence of this vector measure is an element of $\mathfrak{D}^{\cdot 1}(\mathbb{G}, \mathbb{R})$, namely the functional 


$$
\varphi \rightarrow-\int(\mathbf{u} . \nabla \varphi) d \theta
$$

Cobserve that this definition of the divergence of a vector measure does not rely on any connection in the underlying space; it more generally makes sense in the framework of differential manifolds, with vector measures understood as in Sec.7 below).

DEFINITION 3.4. The vector measure $C . \nabla D=u \theta$ is called the masscurrent of the matter in the window $\mathrm{W}$.

The real distribution div $(\mathbf{u} \theta)$ is called the mass-input relative to this window.

A vector distribution, such as $F$ in (3.7), is said to take its values in the subspace $\mathbb{E}$ of $\mathbb{G}$ if $\langle F, \varphi\rangle \in \mathbb{E}$ for every $\varphi \in \mathcal{D}^{1}(\mathbb{G}, \mathbb{R})$. By observing that (div C). $\nabla D=\operatorname{div}(C . \nabla D)$, one obtains:

PROPOSITION 3.5. The vector distribution divC takes its values in $\mathbb{E}$ if and only if the mass-input is zero.

REMARK 3.6. Since the linear space $\mathbb{E}$ is equipped with a Euclidean metric, it makes sense to impose on a Galilean coordinate system, say $0 x^{0} x^{1} x^{2} x^{3}$, the condition of orthonormality in what concerns the "spatial" axes $0 x^{1} x^{2} x^{3}$. Now, one observes that any change of Galilean coordinates preserving this condition is expressed by a matrix whose determinant equals \pm 1 . Consequently, a well defined nonnegative real measure in the space $\mathbb{G}$ may be introduced as admitting the Lebesgue measure of $\mathbb{R}^{4}$ as image in any of these special coordinate systems. We shall call this measure the Galilean volume. Alternatively, if a frame-free construction is wished, one may put $E(t)=\{\xi \in \mathbb{G}: \mathbb{D}(\xi)=t\}$. For every $t \in \mathbb{R}$, this level set of the date function is an affine space, equipped with a Euclidean metric since its vectors let themselves be identified with the elements of $\mathbb{E}$. Hence, in $E(t)$, the three-dimensional volume is frame-free defined, a real measure denoted here by $v_{t}$. Then, the Galilean volume emerges as the element of $\mathfrak{D}^{\circ}(\mathbb{G}, \mathbb{R})$ assigning to every $\varphi \in \mathcal{D}^{0}(\mathbb{G}, \mathbb{R})$ the real number $\int_{\mathbb{R}}\left(\int_{E(t)} \varphi(\xi) d V_{t}(\xi)\right) d t$. 
Here is an example of the use of the Galilean volume measure. Let $\Omega$ be a subset of $\mathbb{G}$, assumed open to $f(x$ the ideas. The characteristic function $\chi_{\Omega}$ makes the density, relative to Galilean volume, of some nonnegative real measure $\omega$. In some usual situations, the gradient $\nabla \omega$, a priori an element of $\mathfrak{D}^{\prime \prime}\left(\mathbb{G}, \mathbb{G}^{\prime *}\right)$, happens to belong to $\mathfrak{D}^{\prime 0}\left(\mathbb{G}, \mathbb{G}^{\prime *}\right)$, i.e it equals a covector measure, concentrated on the boundary $\partial \Omega$. For every vector field $n \in \mathcal{D}^{0}\left(\mathbb{G}, \mathbb{G}^{\prime}\right)$, the real number $\langle\nabla \omega, \eta\rangle$ by definition constitutes the (inward) flux of $\eta$ across $\partial \Omega$. This generalizes the familier situation where $\partial \Omega$ is a smooth surface and gives rise to formulas of the GreenOstrogradsky type; [4] and [22] are reference books on questions of this sort.

REMARK 3.7. Some terms expressing "forces" or "efforts" should naturally contribute in the right-hand member $F \in \mathcal{D}^{\prime 1}\left(\mathbb{G}, G^{\prime}\right)$ of (3.7). A connection then has to be made with the virtual power (or virtual work) formalism under which efforts are commonly treated. In Statics, the possible equilibrium of a mechanical system, relative to some reference space $X$, is investigated. To this end, it is usual to describe every effort through the power it would develop in every motion with smooth velocity field $\eta$. By axiom, this power depends linearly on the "test field" $\eta$. In other words, one defines each effort as a real linear functional on some space $\mathcal{D}^{h}(X, \mathbb{E})$, in fact a covector distribution, element of $\mathcal{D}^{h}\left(X, \mathbb{E}^{*}\right)$. When coming to Dynamics, the definition of efforts has to be expanded in the dimension of time too. Each effort will then appear as an $\mathbb{E}^{*}$-valued distribution in $\mathbb{G}$, in practice an element of $\mathcal{D}^{\prime h}\left(\mathbb{G}, \mathbb{E}^{*}\right)$ for some integer $h$.

In contrast, the distribution $F$ in (3.7) is $G$-valued; more specially, if the mass-input vanishes, this distribution is $\mathbb{E}$-valued. At the present stage, this lack of consistency is readily overcome by observing that the Euclidean metric of $\mathbb{E}$ allows one to identify this space with its dual. The introduction of the operator "equ", instead of "div", in Sec.7 below, will provide a deeper insight. 


\section{A single particle.}

Let $P$ be a punctual particle, with mass $m>0$. Let $p(t)$ denote, as in (3.5), its position -we shall rather say its placement, in accordance with the current terminology of Continuum Mechanics- at time $t$ in the inertial reference space $X$. Let $O x^{1} x^{2} x^{3}$ denote an orthonormal Cartesian frame of this space.

The motion $p: \mathbb{R} \rightarrow X$ is assumed continuous. Equivalently, the mapping $t \rightarrow \pi(t) \simeq(t, p(t))$ is continuous of $\mathbb{R}$ to $\mathbb{G}$. In view of the special form of its first component, it is clear that $\pi$ is injective and proper, in the sense that the inverse image of every compact subset of $\mathbb{G}$ is compact in $\mathbb{R}$.

When, in a problem of Continuum Mechanics, the motion of individuated particles is expressed, one is used to say that investigation is conducted in Lagrange variables. In contrast, the Euler variable standpoint consists in focusing attention on the velocity field in time-space. The formulation of Dynamics we are proposing clearly is of the latter sort. This section is to demonstrate that, nevertheless, the said formulation is able to generate differential equations of the Lagrangian style.

We first have to state the definition of the model "punctual particle" in this framework. Here, the chosen window $W$ will be the whole of $\mathbb{G}$.

The presence measure of the particle in $\mathbb{G}$, an element of $\mathfrak{D}^{\circ}(\mathbb{G}, \mathbb{R})$, is defined as the linear functional $\theta$ which assigns to every $\varphi \in D^{0}(\mathbb{G}, \mathbb{R})$ the real number $\langle\theta, \varphi\rangle=m \int_{\mathbb{R}} \varphi(\pi(t)) d t$. In fact, because every compact subset of $\mathbb{G}$ has a compact inverse image under $\pi$, one readily checks that $\theta$ meets the suitable continuity requirements for being a measure. Since $m>0$, the expression $\langle\theta, \varphi\rangle$ is nonnegative for every nonnegative $\varphi$ (an alternative reason for asserting that $\theta$ is a measure). In other words, if $\ell$ denotes the Lebesgue measure on $\mathbb{R}$, then $\theta$ equals the image under $\pi$ of the measure $\mathrm{ml}$.

There is now to introduce the velocity field $\mathbf{u}$ of the investigated material. The natural assumption to make, in order to allow for its construction, is that the mapping $p$, or equivalently $\pi$, is locally absolutely 
continuous. Then the derivative $\dot{\pi}(t) \simeq(1, \dot{p}(t))$ exists for almost every $t$ and $t \rightarrow \dot{\pi}(t)$ makes an element of $L_{\text {loc }}^{1}\left(\mathbb{R}, l ; \mathbb{G}^{\prime}\right)$. The complementary set $\mathbb{G} \backslash \pi(\mathbb{R})$ is $\theta$-negligible. Hence, for $\theta$-almost every point $\xi$ of $\mathbb{G}$, there exists a unique $t \in \mathbb{R}$ such that $\xi=\pi(t)$. Therefore, four functions $\xi \rightarrow u^{\alpha}(\xi):=\dot{\pi}^{\alpha}\left(\pi^{-1}(\xi)\right)$ are defined $\theta$-a.e. in $\mathbb{G}$, with $u^{\alpha}(\xi) \equiv 1$ if $\alpha=0$ and $u^{\alpha}(\xi)=\dot{p}^{\alpha}\left(\pi^{-1}(\xi)\right)$ otherwise. Through standard properties of the images of measures, the vector field $\mathbf{u}$ which has these components is an element of $L_{l o c}^{1}\left(\mathbb{G}, \theta ; \mathbb{G}^{\prime}\right)$; this vector field more specially belongs to $L_{\text {loc }}^{2}\left(\mathbb{G}, \theta ; \mathbb{G}^{\prime}\right)$ if and only if $\dot{\pi} \in L_{\text {loc }}^{2}\left(\mathbb{R}, l ; \mathbb{G}^{\prime}\right)$ (equivalently $p \in W_{10 \mathrm{c}}^{1,2}(\mathbb{R}, X)$ ).

Under the latter conditions, the kinetic tensor measure $C$ exists; its components $C^{\alpha \beta}=u^{\alpha} u^{\beta} \theta$ are the linear functionals

$$
C^{\alpha \beta}: \varphi \rightarrow m \int_{\mathbb{R}} \dot{\pi}^{\alpha}(t) \dot{\pi}^{\beta}(t) \varphi(\pi(t)) d t .
$$

Due to the definition of partial derivatives in the theory of Distributions, the left-hand member of (2.2) in the present case equals the functional defined, for every $\varphi \in \mathcal{D}^{1}(\mathbb{G}, \mathbb{R})$, by

$$
\left\langle C_{, \beta}^{\alpha \beta}, \varphi\right\rangle=-\left\langle C^{\alpha \beta}, \varphi_{, \beta}\right\rangle=-m \int_{R} \dot{\pi}^{\alpha}(t) \dot{\pi}^{\beta}(t) \varphi_{, \beta}(\pi(t)) d t .
$$

Observe that $t \rightarrow \varphi(\pi(t))$ is an absolutely continuous function, with derivative equal to $\dot{\pi}^{\beta}(t) \varphi_{, \beta}(\pi(t))$ for almost every $t$. Since $\dot{\pi}^{0} \equiv 1$, this yields in particular

$$
\left\langle C^{0 \beta}, \varphi\right\rangle=-m \int_{R} \frac{d \varphi(\pi(t))}{d t} d t=0
$$

1.e. the mass-input is zero. This reflects the implicit assumption that the particle evolves without collecting nor losing any material.

Consequently, in view of Prop. 3.5, the vector distribution divc takes its values in $\mathbb{E}$. For consistency with the writing of Classical Mechanics, we shall, in the rest of the section, identify this Euclidean linear space with its dual.

By the notation $Y \in \operatorname{lbv}(I, \mathbb{E})$, we mean that $Y$ is a function of a real interval I to $\mathbb{E}$ (or, more generally, to a Banach space [16]) with locally bounded variation, i.e. $Y$ has bounded variation on every compact subinterval of $I$. With such a function $Y$, an $\mathbb{E}$-valued measure on $I$ is 
classically associated, that we denote by dy and call the dirrerential measure of $Y$. A measure differential equation [16] [18] is a condition imposed on an unknown $Y \in \operatorname{lbv}(I, \mathbb{E})$ in the form of an equality of $\mathbb{E}$-valued measures

$$
d Y=\Phi(t, Y(t)) d h,
$$

where $d h$ is a given real measure on $I$ and $\Phi: I \times \mathbb{E} \rightarrow \mathbb{E}$ a given function meeting suitable regularity requirements. Because $\mathbb{E}$ has finite dimension (this would more generally hold for a Banach space possessing the Radon-Nikodym property [16]), a nonnegative real measure ds on I is sure to exist (non uniquely), relative to which or and dh possess density functions $Y_{s}^{\prime} \in L_{\text {loc }}^{\prime}(I, d s ; \mathbb{E})$ and $h_{s}^{\prime} \in L_{\text {loc }}^{1}(I, d s ; \mathbb{R})$. Then the above condition is equivalent to an equality in $L_{\text {loc }}^{\prime}(I, d s ; \mathbb{E})$. In particular, the Lebesgue measure on I may be taken as ds if and only if both functions $Y$ and $h$ happen to be locally absolutely continuous. In the latter case, the measure differential equation reduces to a differential equation in the usual (Carathéodory) sense.

Incidentally, for mechanical systems presenting unilateral constraints and/or dry friction, efforts are connected to the motion through relations of such a form that Dynamics is finally expressed in a measure differential inclusions [16] [17].

PROPOSITION 4.1. Suppose that the distribution $F \in \mathcal{D}^{\prime 1}(\mathbb{G}, \mathbb{E})$, expressing the efrorts that the investigated particle experiences. happens to be a measure. Then the fundamental equation (3.7) holds if and only if the element $\dot{p}$ or $L_{\text {loc }}^{2}(\mathbb{R}, \mathbb{E})$ [possesses a representative which] is a function with locally bounded variation, satisfying the measure differential equation

$m d \dot{p}=\pi^{-1}(F)$

Proof. Choose $\psi \in \mathcal{D}(\mathbb{R}, \mathbb{R})$ with support contained in some compact interval I; the image of I under the continuous mapping $p$ is a compact subset, say $K$, of $X$. Denote by $\zeta$ an element of $\mathcal{D}(X, \mathbb{R})$ with value 1 throughout a 
neighbourhood of $K$. Then the function $\varphi$ defined as

$$
\xi \simeq\left(x^{0}, x\right) \rightarrow \varphi(\xi)=\psi\left(x^{0}\right) \zeta(x)
$$

belongs to $\mathcal{D}(\mathbb{G}, \mathbb{R})$. With this choice of $\varphi$, the expression in (4.2) becomes

$$
\left\langle C_{, \beta}^{\alpha \beta}, \varphi\right\rangle=-m \int_{\mathbb{R}} \dot{\pi}^{\alpha}(t) \frac{d \varphi(\pi(t))}{d t} d t=-m \int_{R} \dot{\pi}^{\alpha}(t) \psi^{\prime}(t) d t
$$

Consequently, if (3.7) holds, one has, for every $i \in\{1,2,3\}$,

$$
-m \int_{\mathbb{R}} \dot{p}^{i}(\mathrm{t}) \psi^{\prime}(\mathrm{t}) \mathrm{dt}=\left\langle\mathrm{F}^{i}, \varphi\right\rangle .
$$

If the distribution $F^{i}$ is a measure, there exists a real number $A>0$ such that, for every $\varphi \in \mathcal{D}(\mathbb{G}, \mathbb{R})$ with support contained in the compact subset I $\times \operatorname{supp} \zeta$ of $\mathbb{R} \times X \simeq \mathbb{G}$, the inequality $\left|\left\langle F^{\prime}, \varphi\right\rangle\right| \leqslant A\|\varphi\|_{\infty}$ nolds. Therefore, by choosing $\varphi$ under the form (4.4), one obtains

$$
\left\|\int_{R} \dot{p}^{i}(t) \psi^{\prime}(t) d t \mid \leqslant m^{-1} A\right\| \psi \|_{\infty} \text {. }
$$

for every $\psi$ with support contained in I. This classically entalls that $\dot{p}^{i}$ has locally bounded variation. Furthermore, the right-hand side of (4.5) equals $\left\langle\pi^{-1}\left(F^{i}\right), \psi\right\rangle$. Therefore $\pi^{-1}\left(F^{i}\right)$ equals the derivative, in the sense of the Distributions on $\mathbb{R}$, of the measure $m \dot{p}^{i} \mathrm{dt}$. This derivative is nothing but the differential measure of the function $\dot{p}^{i}$, so (4.3) is established.

Conversely, if $p^{\prime}$ verifies (4.3), the same calculation shows that $\left\langle C^{\alpha \beta}{ }_{\beta}, \varphi\right\rangle=\left\langle F^{\alpha}, \varphi\right\rangle$ for every $\varphi$ of the form (4.4). By density, the same holds for every $\varphi \in \mathcal{D}(\mathbb{G}, \mathbb{R})$.

The most familiar case, where the distribution $F$ can be asserted to equal a measure, is that of a particle submitted to a force field. Giving such a field consists in defining, on a subset of $\mathbb{G}$ assumed at all event to contain $\pi(\mathbb{R})$, a universally locally integrable (for instance continuous) $\mathbb{E}$-valued function, say $\mathrm{f}$. The particle is sald submitted (proportionally to its mass) to the force field if the distribution $F$ equals the $G^{\prime}$-valued measure possessing the function $\xi \rightarrow(0, f(\xi))$ as density, relative to the presence measure $\theta$. In such a case, the measure $\pi^{-1}(F)$ is found to possess the function $t \rightarrow(0, m f(\pi(t)))$ as density relative to the Lebesgue measure $\ell$ of $\mathbb{R}$. Therefore, (4.3) is satisfied if and only if the $\mathbb{E}$-valued measure $d \dot{p}$ possesses $t \rightarrow f(\pi(t))$ as density relative to $l$. This equivalently means 
that $\left.p \in W_{10 c}^{2,1}(\mathbb{R}, X)\right)$ and that the classical dirrerential equation $\ddot{p}(t)=$ $f((t, p(t)))$ holds

In general, as soon as $p \in W_{10 c}^{2,1}(\mathbb{R}, X)$ ), each component $C_{\beta}$ of the vector distribution div $C$ equals a real measure on $\mathbb{G}$; this is the image of the measure $m \ddot{\pi}^{\alpha} l$ under the (continuous, injective and proper) mapping $\pi: \mathbb{R} \rightarrow \mathbb{G}$. Alternatively, this may be established by performing on the expression (4.2) an integration by parts, yielding

$$
\left\langle C^{\alpha \beta}, \beta, \varphi\right\rangle=m \int_{R} \ddot{\pi}^{\alpha}(t) \varphi(\pi(t)) d t .
$$

Essentially $\ddot{\pi}^{0}=0$, while $\ddot{\pi}^{1}, \ddot{\pi}^{2}, \ddot{\pi}^{3}$ equal, for almost every $t$, the components of the acceleration vector $\ddot{p}(t) \in \mathbb{E}$, relative to the reference space $X$.

\section{Initial conditions.}

This Section provides examples of the change of window through the restriction procedure. For simplicity, let us assume that the analysis of some dynamical situation has been first conducted with the whole space $\mathbb{G}$ used as window and that the corresponding distribution $F$ in the fundamental equation has been found to be a $\mathbb{G}^{\prime}$-valued measure. As a part of the proposed formulation of Dynamics, we now are going to stipulate how the elements of this analysis relate to the treatment of Dynamics one could alternatively conduct when using another window $\mathbb{W}$. The latter will be supposed to be a Borel subset of $\mathbb{G}$, so that its characteristic function, say $X$, belongs to $L^{\infty}$ of any (Radon) measure.

Let us respectively denote by $\theta, \mathbf{u}, \boldsymbol{c}$ the presence measure, the velocity field and the kinetic tensor measure in the former treatment. We decree that, when the window $\mathrm{w}$ is used, the presence measure should be $\theta_{*}=x \theta$ and the velocity field $u_{*}=x u$. Consequently, the kinetic tensor measure equals $C_{*}=u_{*} \otimes u_{*} \theta_{*}=x C$. The fundamental equation always has the form of an equality of distributions in $\mathbb{G}$. In view of what has been previously written with $\mathbb{G}$ as window, the new equation necessarily is

$$
\operatorname{div} C_{*}=X F+\operatorname{div}(X C)-\chi \operatorname{div} C \text {. }
$$


The products $X F$ and $X$ divC make sense because, by assumption, $F$ and, consequently, divc are measures.

One naturally interprets $X F$ as the part of the formerly considered right-hand member $\mathrm{F}$ which is "visible" in the window w.

The vector distribution $\operatorname{div}(X C)-\chi \operatorname{div} C$ is easily found to have its support contained in the boundary of $W$. This distribution conveys some information about what happens outside the restricted window one is now using. Additional regularity assumptions may confer to this term a more suggestive look.

Suppose, for instance, that $C$ has the form $C_{\gamma}^{\prime} \gamma$, where $\gamma$ denotes the Galilean volume (Rem. 3.6) and $C_{\gamma}^{\prime}$ an element of $L_{\text {loc }}^{1}\left(\mathbb{G}, \gamma ; \mathbb{G}^{\prime} \otimes_{\mathrm{s}} \mathbb{G}^{\prime}\right)$. Suppose that the latter tensor function has locally bounded variation, in the four-dimensional sense [4][22] and that $W$ is closed, with boundary $\partial W$ equal to a $c^{2}$ hypersurface. Then, the real measure $-x \gamma$ possesses as distribution gradient a $\mathbb{G}^{* *}$-valued measure, say $\mathbf{v}$, with support contained in $\partial W$, which may be viewed as the outgoing flux operator, relative to $W$. The above assumptions secure that $\operatorname{div} C$ is a $\mathbb{G}^{\prime}$-valued measure and that $C_{\gamma}^{\prime}$ possesses an outside trace on aw, say $\mathrm{C}_{\gamma}^{+}{ }^{+}$, which is a locally $v$-integrable tensor function. One finally obtains that, in such a case, the term $\operatorname{div}(X C)-X \operatorname{div} C$ equals $C_{\gamma}^{\prime+}, v$ (the dot refers to contracted tensor product).

The variables $t$ and $x^{0}$, in Sec. 4, were assumed to range through the whole of $\mathbb{R}$. Problems pertaining to a limited time interval may be formulated as well, provided the window is restricted adequately.

EXAMPLE 5.1. As the first example, let $W$ equal the closed half space

$$
\overline{\mathbb{F}}\left(t_{0}\right)=\left\{\xi \in \mathbb{G}: \mathbb{D}(\xi) \geqslant t_{0}\right\},
$$

which may be called the closed future of instant $\mathrm{t}_{0}$. By this choice we do not mean that the concerned mechanical system was not in existence before $\mathrm{t}_{0}$, but that investigation begins at this instant.

We shall restrict ourselves, for brevity, to the framework of Sec. 4 , 
1.e. the system consists of a single particle, with motion described by $\pi \in W_{10 c}^{1,2}(\mathbb{R}, \mathbb{G})$ and we assume that the distribution $F$ is a measure. $50 \dot{\pi}$ is an element of $\operatorname{lbv}\left(\mathbb{R}, \mathbb{G}^{3}\right)$; this implies the existence of $\dot{\pi}^{-}\left(t_{0}\right)$ and $\dot{\pi}^{+}\left(t_{0}\right)$, the limits of the absolute velocity $\dot{\pi}$ on the left and on the right of $t_{0}$, denoted in the sequel by $\mathbf{u}_{0}^{-}$and $\mathbf{u}_{0}^{+}$.

Let $\chi_{0}$ denote the characteristic function of the subset $\bar{F}\left(t_{0}\right)$ of $\mathbb{G}$. In terms of the presence measure $\theta$ introduced in Sec. 4 , the presence measure relative to the new window equals $\chi_{0} \theta$, i.e. the functional

$$
\theta_{0}: \varphi \rightarrow m \int_{t \geqslant t_{0}} \varphi(\pi(t)) d t .
$$

The kinetic tensor measure, in the former treatment, was $C=\mathbf{u} \otimes \mathbf{u} \theta$. In the new window, it becomes $C_{0}=u \otimes u \theta_{0}=x_{0} C$. Similarly, the vector measure $F$ expressing the forces involved, has to be replaced by $F_{0}=x_{0} F$. In particular, if $F$ expresses the action of a force field $f: \mathbb{G} \rightarrow \mathbb{E}$, one has $F=(0, f) \theta$, thus $F_{0}=(0, f) \theta_{0}$.

Recall that $C_{0}$ and $F_{0}$ must still be considered as distributions on the whole of $\mathbb{G}$. In that sense, let us calculate the components of div $C_{0}$, i.e. the distributions

$$
C_{0}^{\alpha \beta}: \varphi \rightarrow-\left\langle C_{0}^{\alpha \beta}, \varphi_{, \beta}\right\rangle=-m \int_{t \geqslant t_{0}} \dot{\pi}^{\alpha}(t) \dot{\pi}^{\beta}(t) \varphi_{, \beta}(\pi(t)) d t=-m \int_{t \geqslant t_{0}} \dot{\pi}^{\alpha}(t) d(\varphi \circ \pi)(t) .
$$

We now apply a formula for the differential measure of the product of two functions $g$ and $h$ belonging to $\operatorname{lbv}(\mathbb{R}, \mathbb{R})$, namely $[16]$

$$
d(g h)=g^{-} d h+h^{+} d g .
$$

Take $g=\dot{\pi}^{\alpha}$ and $h=\varphi \circ \pi$; since $\varphi \circ \pi$ is locally absolutely continuous, the (at most countable) set of the discontinuity points of $\dot{\pi}^{\alpha}$ is negligible relatively to the measure $d(\varphi \circ \pi)$. Therefore

$$
\dot{\pi}^{\alpha} \mathrm{d}(\varphi \circ \pi)=\left(\dot{\pi}^{\alpha}\right)^{+} \mathrm{d}(\varphi \circ \pi)=\mathrm{d}\left(\dot{\pi}^{\alpha} \times(\varphi \circ \pi)\right)-(\varphi \circ \pi) d \dot{\pi}^{\alpha} .
$$

Besides, for any function $q \in \operatorname{lbv}(\mathbb{R}, \mathbb{R})$ and for any compact interval $\left[t_{0}, t_{1}\right]$, one has

$$
\int_{\left[t_{0}, t_{1}\right]} d q=q^{+}\left(t_{1}\right)-q^{-}\left(t_{0}\right)
$$


Therefore, the distribution $\left(\operatorname{div} C_{0}\right)^{\alpha}$ consists in the functional

$$
\left\langle C_{0, \beta}^{\alpha \beta}, \varphi\right\rangle=m\left(\dot{\pi}^{\alpha}\right)^{-}\left(t_{0}\right)(\varphi \circ \pi)\left(t_{0}\right)+m \int_{t \geqslant t_{0}}(\varphi \circ \pi)(t) d \dot{\pi}^{\alpha}(t) .
$$

This equals the sum of the two following measures:

$1^{\circ}$ a measure concentrated in $W$ which, in view of (4.2), in nothing but the component of rank $\alpha$ of $F_{0}=\chi_{0} F$,

$2^{\circ}$ the point measure with mass $m u_{0}^{\alpha-}$, placed at point $\pi\left(t_{0}\right)$.

In conclusion, if the dynamics of the particle is treated in the window $\bar{F}\left(t_{0}\right)$, one has to retain, of the effort measure $F$ formerly considered, only what is "visible in the window", namely $F_{0}$, and to add to it the $\mathbb{G}^{-}$-valued point measure located at the point with coordinates $\left(\mathrm{t}_{0}, \mathrm{p}^{1}\left(\mathrm{t}_{0}\right)\right.$, $p^{2}\left(t_{0}\right), p^{3}\left(t_{0}\right)$ ) and whose value has components $m$, $m \dot{p}^{1-}\left(t_{0}\right), m \dot{p}^{2-}\left(t_{0}\right)$, $m p^{3-}\left(t_{0}\right)$. Giving the latter measure amounts to specifying the following data: the initial position of the particle, its mass $m$ and the limit $\mathbf{u}_{0}^{-}$of its velocity on the left of $t_{0}$.

Observe that, with regard to the chosen window, the mass-input distribution is no more zero. It equals the real measure with value $m$, located at the point $\pi\left(t_{0}\right) \simeq\left(t_{0}, p\left(t_{0}\right)\right)$.

In the above treatment, the hyperplane $\left\{\xi \in \mathbb{G}: \mathbb{D}(\xi)=\mathrm{t}_{0}\right\}$ is part of the window. If, in particular, the particle experiences a shock at instant $t_{0}$. this will be included in the study, entailing for the right-limit $\mathbf{u}_{0}^{+}$of the absolute velocity a value different from the given left-limit $\mathbf{u}_{0}^{-}$. Here is an alternative viewpoint.

EXAMPLE 5.2. We now take as window the open future of $t_{0}$, i.e.

$$
\mathbb{F}\left(t_{0}\right)=\left\{\xi \in \mathbb{G}: \mathbb{D}(\xi)>t_{0}\right\} .
$$

In that case, a possible shock at instant $t_{0}$ is no part of the study, so giving $\mathbf{u}_{0}^{-}$would not provide sufficient information about the particle history for predicting its further evolution.

Let us denote by $x_{1}$ the characteristic function of $F\left(t_{0}\right)$. The presence measure is now $\theta_{1}=\chi_{1} \theta$, the kinetic tensor measure $C_{1}=\mathbf{u} \otimes \mathbf{u} \theta_{1}$ and the 
effort measure $F_{1}=\chi_{1} F$.

In the place of (5.1), the formula

$$
\int_{\left.\mathrm{t}_{0}, \mathrm{t}_{1}\right]} d q=\mathrm{q}^{+}\left(\mathrm{t}_{1}\right)-\mathrm{q}^{+}\left(\mathrm{t}_{0}\right)
$$

has to be used, yielding instead of (5.2),

$$
\left\langle C_{0, \beta}^{\alpha \beta}, \varphi\right\rangle=m\left(\dot{\pi}^{\alpha}\right)^{+}\left(t_{0}\right)(\varphi \circ \pi)\left(t_{0}\right)+m \int_{t>t_{0}}(\varphi \circ \pi)(t) d \dot{\pi}^{\alpha}(t) .
$$

The conclusion is analogous to what has been obtained in the preceding case. Dynamics now is expressed by equalling the distribution div $C_{1}$ to the sum of the following terms:

$1^{\circ}$ The vector measure $F_{1}$,

$2^{\circ}$ The point measure with value $m \mathbf{u}_{0}^{+}$, located at $\pi\left(t_{0}\right)$.

\section{Confinement by a boundary.}

Again, in this section, we shall restrict ourselves to the dynamics of a single particle and the time variable will be assumed to range through the whole of $\mathbb{R}$. The effect of a material boundary, that the particle is not allowed to cross, will be taken into account.

Thereby, the mapping $\pi$ is permitted to take only values in some region of $\mathbb{G}$. Let this region be defined by an inequality $b(\xi) \leqslant 0$, with given $b \in C^{2}(\mathbb{G}, \mathbb{R})$, so the boundary is described as the hypersurface $S$ of $\mathbb{G}$, with equation $b(\xi)=0$. The section of $S$ by an isochronous hyperplane $\{\xi: \mathbb{D}(\xi)=t\}$, is denoted by $S_{\mathrm{t}}$. In order that all these sections properly represent boundaries at the corresponding instants, one assumes that, at every point of $S$, the gradient $\nabla b$ does not belong to the linear subspace generated in $\mathbb{G}^{\prime}$ by $\nabla \mathbb{D}$ (in particular, it does not vanish).

In that way, only the global location of the boundary in time-space is given, without any further information about the motion of the material it is made of. Such a description would clearly not be enough if one attempted to take into account any frictional ef fect occurring in the event of contact. But it will prove sufficient for developing the model of an ideal boundary, 
through which the following physical assumptions, concerning the effort that the boundary possibly exerts on the conf ined particle, are formalized:

$1^{\circ}$ The effort vanishes on any time interval during which the particle does not touch the boundary.

$2^{\circ}$ In the event of contact, no adhesion, l.e. glueing or welding effect, takes place.

$3^{\circ}$ In the same event, no friction is present.

DEFINITION 6.1. The hypersurface $S$ is said to constitute an ideal boundary if the feasibility of a distribution $R \in \mathcal{D}^{\prime 1}(\mathbb{G}, \mathbb{E})$, for expressing the effort it exerts upon the confined material (namely the particle, in the present instance) is characterized by the following property:

For every $\eta \in \mathcal{D}^{\prime}(\mathbb{G}, \mathbb{E})$ which, at any point of $S$, satisfies $n . \nabla b \geqslant 0$, one has $\langle R, n\rangle \leqslant 0$.

Here is a consequence of this property.

PROPOSITION 6.2. Every $R \in \mathcal{D}^{\prime 1}(\mathbb{G}, \mathbb{E})$ agreeing with the above Definition is an $\mathbb{E}$ - valued measure on $\mathbb{G}$ with support contained in $\mathrm{S}$. For every representation of this measure in the form $R=R_{j}^{\prime} \mu$, where $\mu$ denotes a nonnegative real measure on $\mathbb{G}$ and $R_{\nu}^{\prime} \in L_{\text {loc }}^{1}(\mathbb{G}, \mu ; \mathbb{E})$, the value $R_{\nu}^{\prime}(\xi)$ is, at $\mu$-almost every point $\xi$, an element of $\mathbb{E}$ orthogonal to $S_{D(\xi)}$ and directed toward the permitted region.

Proof. We shall restrict the proof to the special case where $S$ equals a hyperplane of $\mathbb{G}$. In fact, the techniques presented in Sec.7 below, concerning vector distributions on manifolds and their representation in arbitrary coordinates, make it possible to reduce the general case to this one.

In this special case, a Galilean coordinate system of $\mathbb{G}$, in the sense of Definition 3.3, may be chosen such that the permitted region equals the half-space $x^{1} \leqslant 0$. We thus take as $b$ the linear function $\xi \rightarrow x^{1}$, so $\nabla b$ equals the constant vector with components $(0,1,0,0)$. The components $R^{i}$, $i \in\{1,2,3\}$, of the vector distribution $R$ equal, by definition, the elements of 
$\mathcal{D}^{\prime}(\mathbb{G}, \mathbb{R})$ such that, for every $\eta \in \mathcal{D}^{1}(\mathbb{G}, \mathbb{E})$, one has $\langle R, \eta\rangle=\left\langle R_{i}, \eta^{i}\right\rangle$. The condition $\eta . \nabla b \geqslant 0$ on $S$ reduces, in the present case, to $\eta^{1} \geqslant 0$ holding throughout the coordinate hyperplane $x^{1}=0$. Since this imposes no restriction on $\eta^{2}$ and $\eta^{3}$, one concludes that the distributions $R_{2}$ and $R_{3}$ vanish. As for the distribution $R_{1}$, it equals the linear functional which assigns to every $\eta^{1} \in \mathcal{D}^{1}(\mathbb{G}, \mathbb{R})$ the real number $\left\langle R, \eta^{1} \mathbf{i}_{(1)}\right\rangle$. First, the assumption made implies that this real number should be $\leqslant 0$ as soon as $\eta^{\prime}$ is a nonnegative element of $\mathcal{D}^{1}(\mathbb{G}, \mathbb{R})$, classically implying that $R_{1}$ equals a nonpositive real measure. Secondly, this assumption entails that such a functional assigns the value zero to every $\eta^{1}$ whose support does not intersect $S$, so the support of $R$ is contained in $S$. The asserted properties follow, through standard arguments.

To fix the ideas, let us assume that, in addition to the above boundary efforts, the investigated particle is submitted to a given force field. Then, according to Prop. 4.1 , the velocity function $t \rightarrow \dot{\pi}(t)$ belongs to $\operatorname{lbv}\left(\mathbb{R}, \mathbb{G}^{\prime}\right)$. However, assuming that the boundary fullfills the requirement of Definition 6.1 is a priori not enough to secure that the function $\pi$ takes its values in the permitted region only. We now are going to show how adequate window restriction allows one to take this requirement into account.

Let us use as window the permitted region $W=\{\xi \in \mathbb{G}: b(\xi) \leqslant 0\}$. Asserting that the fundamental equation (3.7) is satisfied with $F$ equal, in view of what precedes, to an $\mathbb{E}$-valued distribution on $\mathbb{G}$, implies, through Prop. 3.5, that the corresponding mass-input vanishes. Then the following Proposition may be invoked.

PROPOSITION 6.3. Assume that, relatively to the above window, the motion $\pi \in W_{\text {loc }}^{1,2}(\mathbb{R}, \mathbb{G})$ has zero mass-input, but nonzero presence measure. Then $\pi(\mathrm{t})$ belongs for every $\mathrm{t}$ to the permitted region.

Proof. Put $w:=\{t \in \mathbb{R}: \pi(t) \in W\}$. The mass current equals the vector measure

$$
\varphi \rightarrow m \int_{t \in W} \dot{\pi}(t) \varphi(\pi(t)) d t=m \int_{t \in \mathbb{R}} x_{w}(t) \dot{\pi}(t) \varphi(\pi(t)) d t,
$$


where $x_{w}$ denotes the characteristic function of $w$. The mass-input is the divergence of this vector measure, namely the distribution

$$
\varphi \rightarrow-m \int_{t \in \mathbb{R}} \chi_{w}(t) \dot{\pi}^{\alpha}(t) \varphi_{, \alpha}(\pi(t)) d t=-m \int_{t \in \mathbb{R}} x_{w}(t) \frac{d}{d t} \varphi(\pi(t)) d t .
$$

Choose I, $\psi, \zeta, \varphi$ in the same way as in the proof of Prop. 4.1. This yields

$$
\forall \psi \in \mathcal{D}(\mathbb{R}, \mathbb{R}): \int_{t \in \mathbb{R}} x_{w}(t) \psi^{\prime}(t) d t=0,
$$

meaning that $\chi_{w}$ has zero derivative in the sense of the Distributions. Therefore, this function takes a constant value $B$ (equal to 0 or 1 ), except possibly in some Lebesque-negligible subset of $\mathbb{R}$.

Since $W$ is closed in $G$, the function $X_{w}$ is U.S.C. on $\mathbb{R}$. Imagine the existence of $\tau \in \mathbb{R}$ whith $\chi_{w}(\tau)=0$; then $\chi_{w}$ vanishes on a neighbourhood of $\tau$. This requires $B=0$, implying that $X_{w}$ vanishes Lebesque-a.e, in contradiction with the assumption of nonzero presence mesure relatively to the window $W$.

REMARK 6.4. Using Prop. 4.1, one finds that, under the above conditions, the velocity function $t \rightarrow \dot{\pi}(t)$ belongs to $\operatorname{lbv}\left(\mathbb{R}, G^{\prime}\right)$ and that the dynamics of the particle is governed by a measure differential inclusion [12][17]. But, in the event of the particle colliding with the boundary, evolution is not uniquely determined by this inclusion.

Actually, the circumstances of shocks are, in practice, so complex that the physical information needed for a deterministic analysis is usually out of reach.

If the boundary is fixed (relative to some inertial reference space), it is traditional to complement the statement of evolution problems by the requirement of energy preservation. The possible collisions are then called elastic bounces. Even so, the uniqueness of solution to initial value problems is conditioned by additional smoothness assumptions [2][19][21]. Energy balance for motions with lbv velocity function is drawn in [17] and objections to the preservation of (mechanical) energy are raised, even if the materials of which the boundary and the investigated system are made may be treated as perfectly elastic. Preference is given, in [17], to 
the sortness (i.e. inelasticity) assumption of the possible collisions. In the present context, this would be expressed by asserting that, at any $\mathrm{t}$ such that $\mathrm{b}(\pi(\mathrm{t}))=0$, the right-side limit $\dot{\pi}^{+}(\mathrm{t})$ is a tangent vector to $\mathrm{S}$. The resulting evolution problems are dissipative and prove more comfortable, analytically and numerically, than in the case of elastic bounces. Furthermore, the physical circumstances in which the softness assumption may be accepted seem easier to identify in practice. The existence of solutions to the corresponding initial value problems is established in $[8][9]$

\section{Operators def and equ on a Riemannian manifold.}

Three integers $d, r, h$ will recurrently appear in the rest of this paper. Let it be assumed once for all that

$$
0 \leqslant h \leqslant r-1 \leqslant d-2 \text {. }
$$

Let $\mathcal{M}$ denote a $C^{d}$-differential manifold (without boundary), of finite dimension $n$. A Riemannian metric is defined on $\mathcal{M}$ by giving some symmetric doubly covariant tensor field $g$, assumed to be $r$ times continuously differentiable. This we shall express by writing $g \in C^{r}\left(\mathcal{M}, \mathcal{M}^{\prime *} \otimes_{\mathrm{s}} \mathcal{M}^{\prime *}\right)$, in a somehow abusive system of notations, to be applied in all the sequel. Understand that $g$ is actually not a mapping of $\mathcal{M}$ to a set which would be denoted by $\mathcal{M}^{\prime *} \otimes_{\mathrm{s}} \mathcal{M}^{\prime *}$, but a selector, assigning to every $x \in \mathcal{M}$ an element of $\mathcal{M}_{x}^{* *} \otimes_{s} \mathcal{M}_{x}^{* *}$, where $\mathcal{M}_{x}^{* *}$ denotes the cotangent space to $\mathcal{M}$ at point $\mathrm{x}$.

For every vector field $v \in C^{h+1}\left(\mathcal{M}, \mathcal{M}^{\prime}\right)$, the Lie derivative $L_{v} g$ makes sense. This is a $C^{h}$ field, of the same tensorial type as $g$.

DEFINITION 7.1 The differential operator

$$
\text { def: } C^{h+1}\left(\mathcal{M}, \mathcal{M}^{\prime}\right) \rightarrow C^{h}\left(\mathcal{M}, \mathcal{M}^{\prime *} \otimes_{s} \mathcal{M}^{\prime \prime \prime}\right)
$$

defined as

$$
\text { def } v:=\frac{1}{2} L_{v} g
$$

is called the deformation operator. 
This denomination is suggested by the Kinematics of Continua. In fact, if $v$ is the velocity field of a continuous medium in motion throughout $\mathcal{M}$, then def $v$ equals the rate of deformation tensor of the medium (we shall come back to this in Sec.9; see also [7], Chap. 1).

When coordinates are used in $\mathcal{M}$, the classical expression of Lie derivatives [7] yields the components of the tensor def $v$ in the form

$$
(\text { def } v)_{i j}=\frac{1}{2}\left(g_{i j, k} v^{k}+g_{k j} v^{k},+g_{i k} v_{, j}^{k}\right)
$$

Let us now introduce the space $\mathcal{D}^{\text {h }}\left(\mathcal{M}, \mathcal{M}^{\prime} \otimes_{5} \mathcal{M}\right)$ of the tensor distributions on $\mathcal{M}$, of order $\mathrm{h}$ (more correctly, one should say "of order less than or equal to $\mathrm{h}^{\prime)}$, doubly contravariant and symmetric. By definition, an element $T$ of this space is a real continuous linear functional on $\mathcal{D}^{\mathrm{h}}\left(\mathcal{M}, \mathcal{M}^{\prime *} \otimes_{s} \mathcal{M}^{* *}\right)$; the latter denotes the subspace of $C^{h}\left(\mathcal{M}, \mathcal{M}^{* *} \otimes_{5} \mathcal{M}^{* *}\right)$ consisting of fields whose support is compact in $\mathcal{M}$. Continuity is meant in a sense similar to that of the theory of real distributions with order $h$ in $\mathbb{R}^{n}$. In this theory, for every compact subset $K$ of $\mathbb{R}^{n}$, a Banach norm is constructed on the subspace $\mathcal{D}_{K}^{h}\left(\mathbb{R}^{n}, \mathbb{R}\right)$ of $\mathcal{D}^{h}\left(\mathbb{R}^{n}, \mathbb{R}\right)$ consisting of the functions whose support is contained in $K$. This construction involves the suprema of the absolute values of the partial derivatives, up to order $h$, of the considered function. To do the same here, one has to use local charts in $\mathcal{M}$; a compact subset $K$ of $\mathcal{M}$ is covered by the domains of a finite number of them. The norm of an element of $\mathcal{D}_{\mathrm{K}}^{\mathrm{h}}\left(\mathcal{M}, \mathcal{M}^{\prime *} \otimes_{\mathrm{S}} \mathcal{M}^{\prime *}\right)$ is constructed from the partial derivatives, up to order $h$, of its components. It is found that any change of charts replaces the constructed norm by an equivalent one. A functional $\mathcal{D}^{h} \rightarrow \mathbb{R}$ is said continuous if its restriction to every $\mathcal{D}_{K}^{h}$ is continuous.

We shall uniformly denote by $\langle.,$.$\rangle all the \mathbb{R}$-valued bilinear forms corresponding to the pairing of a space of fields with a space of distributions. In contrast, for every $x \in \mathcal{M}$, the pairing bilinear form of $\mathcal{M}_{x}^{\prime}$ and $\mathcal{M}_{x}^{\prime *}$ is denoted by a simple dot, as well as the forms which pair the adequate couples of tensor products of them.

Relatively to a local chart of $\mathcal{M}$, the tensor distribution $T$ is repre- 
sented by its components $T^{i j}$. The latter may be viewed as real distributions on the domain $D$ of the chart (an open submanifold of $\mathcal{M}$ ) or, alternatively, as real distributions on the range of this chart (an open subset of $\mathbb{R}^{n}$ ). From the first standpoint, one may define $T^{i j}$ as follows. For every $x \in D$, the chart induces a base, say $\left\{\mathbf{e}^{(1)}, \ldots, \mathbf{e}^{(n)}\right\}$, in the cotangent space $\mathcal{M}_{x}^{\prime *}$ to $\mathcal{M}$ at point $x$; the assignments $x \rightarrow e^{(i)}$ are $C^{d-1}$ fields of covectors in $D$. Then the element $T^{i j}$ of $\mathcal{D}^{\text {hh }}(D, \mathbb{R})$ is introduced by

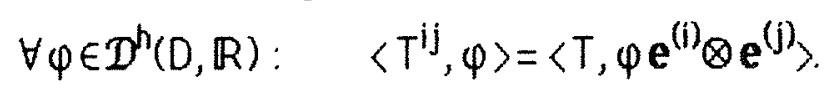

As a consequence, for every $x \in \mathcal{D}^{h}\left(\mathcal{M}, \mathcal{M}^{\prime *} \otimes_{s} \mathcal{M}^{* *}\right)$, one finds $\langle T, \mathcal{X}\rangle=\left\langle\mathrm{T}^{i j}, x_{i j}\right\rangle$. of course, all what precedes can be more generally done for arbitrary tensorial types, without the restriction of symmetry; some other cases will be met in the sequel. This is modelled on De Rham's theory of Currents on differential manifolds [20]; the same concepts have already been used by A. Lichnerowicz [6] [5].

In particular, when $h=0$, the tensor distribution is said to be a doubly contravariant symmetric tensor measure. Its components are real measures in the standard sense. By applying to them the Radon-Nikodym theorem, one shows the existence of a (non unique) nonnegative real measure $\mu$ on $\mathcal{M}$, relative to which $T$ possesses a density $T_{\nu}^{\prime} \in L^{\infty}\left(\mathcal{M}, \mu ; \mathcal{M} \mathcal{M}^{\prime} \otimes_{\mathrm{s}} \mathcal{M} \mathcal{M}^{\prime}\right)$; notation: $T=$ $T_{\mu}^{\prime} \mu$. Therefore, the action of $T$ may in that case be expressed as an integral (7.4)

$$
\langle\mathrm{T}, x\rangle=\int x \cdot \mathrm{dT}=\int x \cdot \mathrm{T}_{\nu}^{\prime} \mathrm{d} \mu=\int x_{\mathrm{ij}} \mathrm{T}_{\mu}^{\mathrm{ij}} \mathrm{d} \mu .
$$

This integral more generally makes sense for every $x \in L^{1}\left(\mathcal{M}, \mu ; \mathcal{M}^{* *} \otimes_{\mathrm{s}} \mathcal{M}^{* *}\right)$. By the notations $L^{1}$ or $L^{\infty}$ here we mean that, in any chart, the components of the considered fields are elements of spaces of the corresponding sorts.

observe that the tensor measures so defined on the manifold $\mathcal{M}$ can by no means be viewed as $\sigma$-additive functions of subsets, except of course for the tensorial order zero, i.e. the case of scalar measures.

DEFINITION 7.2. The negative transpose of def, a linear mapping of $\mathcal{D}^{\text {hh }}\left(\mathcal{M}, \mathcal{M}^{\prime} \otimes_{5} \mathcal{M}^{\prime}\right)$ to $\mathcal{D}^{\text {ht+1 }}\left(\mathcal{M}, \mathcal{M}^{* *}\right)$, is called the equilibrium operator and denoted by equ. In other words, for every $T$ in $\mathcal{D}^{\text {hh }}\left(\mathcal{M}, \mathcal{M}^{\prime} \otimes_{\mathrm{S}} \mathcal{M}^{\prime}\right)$ and every $\eta$ in $\mathcal{D}^{h+1}\left(\mathcal{M}, \mathcal{M}^{\prime}\right)$. 
The denomination "equ" is suggested by the treatment of the Statics of a Continuous Medium through the Method or Virtual Power: in this method, some test velocity fields, similar to $\eta$ above, are considered. Assume that the tensor distribution $T$ is meant to represent the internal efforts of the medium, in such a way that, whenever the medium moves with $\eta$ as velocity field, $\langle T$, def $\eta\rangle$ equals the power of these efforts. Similarly, assume that the external efforts are represented by an element $F$ of $\mathcal{D}^{h+1}\left(\mathcal{M}, \mathcal{M}^{\prime *}\right)$, i.e. the corresponding power equals $\langle F, n\rangle$. Then, the requirement of zero total power for every test field $\eta$ yields the equilibrium equation of the medium in the form $F=$ equ $T$.

If coordinates are used in $\mathcal{M}$, one derives from (7.2) and (7.5) the expression of the components of equ $T$ :

$$
\text { (equT })_{k}=g_{j k}{ }^{i j}{ }_{, i}+\left(g_{i k, j}-\frac{1}{2} g_{i j, k}\right) T^{i j} \text {. }
$$

The products of distributions by functions, which appear on the right-hand side, make sense, due to Inequalities (7.1).

REMARK 7.3. If the Riemannian manifold $\mathcal{M}$ simply consists in a Euclidean space, one readily finds, by using orthonormal Cartesian coordinates and dropping the distinction between covariance and contravariance, that the operator "equ" coincides with what, in Secs. 2 and 3 , has been introduced as the "divergence" of the considered tensor distribution. The meaning that the latter operator may take in the manifold context calls for some comments; also the case of tensor fields has to be compared with that of tensor distributions.

Let us agree [7] to mark with " the subscript introduced by the covariant derivation of any differentiable field, relatively to the standard torsion-free connection associated with the Riemannian metric. In particular, the divergence of a (doubly contravariant) tensor field $\Phi \in C^{\prime}\left(\mathcal{M}, \mathcal{M}^{\prime} \otimes \mathcal{M}^{\prime}\right)$ is defined as the (contravariant) vector field with components $(\operatorname{div} \Phi)^{\mathrm{j}}=\Phi_{\|}^{\mathrm{ij}}$. Here $\Phi$ is not necessarily symmetric and this 
more generally applies to a tensor field of higher rank, provided its first index corresponds to contravariance. In [6], the negative of this operation of contracted covariant derivation is called codifferentiation.

The operation ${ }_{i}$ may also be introduced for a tensor distribution, element of some space of the $\mathcal{D}^{\text {hh }}$ sort. This is, by definition, the negative transpose of the similar operation, applied to fields belonging to the paired $\mathcal{D}^{h}$ space. The covariant derivative, associated in that way with any tensor distribution, is another tensor distribution of one unit up in tensorial rank and one unit up in distributional order. It obeys the same calculation rules as the covariant derivative of tensor fields. Its construction is indifferent to the choice of a chart: this is made clear by observing that the operator so defined on some $\mathcal{D}^{\text {hh }}$ space equals the negative transpose of the divergence operator, defined on the adequate $\mathcal{D}^{\mathrm{h}+1}$ space.

Covariant derivation allows one, in particular, to introduce the divergence of a tensor distribution $T \in \mathcal{D}^{\mathrm{h}}\left(\mathcal{M}, \mathcal{M}^{\prime} \otimes \mathcal{M}\right)$ as the element $\operatorname{div} T$ of $\mathcal{D}^{\mathrm{h}+1}(\mathcal{M}, \mathcal{M})$ such that (div $\left.T\right)^{\mathrm{j}}=\mathrm{T}^{\mathrm{ij}}{ }_{\text {ii }}$.

As another instance, the covariant derivative of a real measure $\mu \in \mathcal{D}^{.0}(\mathcal{M}, \mathbb{R})$ equals the element $\nabla y$ of $\mathcal{D}^{.1}\left(\mathcal{M}, \mathcal{M}^{* *}\right)$, with components $\bigcup_{\| i} \in \mathcal{D}^{\prime 1}(\mathcal{M}, \mathbb{R})$ such that

$$
\forall n \in \mathcal{D}^{\prime}\left(\mathcal{M}, \mathcal{M}^{\prime}\right):\left\langle\eta^{i}, \varphi_{\mid i}\right\rangle=-\left\langle\eta_{\mid i}^{i}, y\right\rangle .
$$

The Riemannian volume is the nonnegative real measure $\rho$ on $\mathcal{M}$ equal, as soon as an arbitrary local chart $(x)$ is chosen, to $0=\left(\operatorname{det} g_{(x)}\right)^{1 / 2} l_{(x)}$; here $l_{(x)}$ denotes the real measure on $\mathcal{M}$ whose image in the chart equals the Lebesgue measure of $\mathbb{R}^{n}$. One finds $\rho_{\| i}=0$.

For $\Phi \in C^{1}\left(\mathcal{M}, \mathcal{M}^{\prime} \otimes \mathcal{M}\right)$ as above, $\Phi \rho$ is an element of $\mathcal{D}^{\circ}\left(\mathcal{M}, \mathcal{M}^{\prime} \otimes \mathcal{M}^{\prime}\right)$ and it turns out that $\operatorname{div}(\Phi \rho)=(\operatorname{div} \Phi) \rho$.

In contrast with divT, the element equT, as defined by (7.5), is a covector distribution. Also recall that (7.5) essentially applies to symmetric $T$ (equivalently, if this definition is used for nonsymmetric $T$, the result depends only on the symmetric part of it). By observing that, for every differentiable vector field $\eta$, one has [7], 


$$
(\text { def } \eta)_{i j}=\frac{1}{2}\left[\left(g_{k i} \eta^{k}\right)_{1 j}+\left(g_{k j} \eta^{k}\right)_{1 i}\right]
$$

one derives from (7.5) that, for $T \in \mathcal{D}^{\text {hh }}\left(\mathcal{M}_{,} \mathcal{M}^{\prime} \otimes_{5} \mathcal{M}^{\prime}\right)$,

$$
(\text { equ } T)_{i}=g_{i j}(\operatorname{div} T)^{j} \text {. }
$$

\section{Lagrange equations.}

The setting of this section is the Analytical Dynamics of a mechanical system with finite freedom $n$. Classically, the system possible states constitute a $C^{r}$-differential manifold $Q$, where some local coordinates are denoted by $q^{1}, \ldots, q^{n}$. For simplicity, we shall make the scleronomy assumption, i.e. the constraints underlying the above parametrization do not depend on time. Consequently, the generic expression of the kinetic energy is a time-independent positive definite quadratic form with respect to the time-derivatives $\dot{q}^{\prime}$, say

$$
E_{c}=\frac{1}{2} g_{i j}(q) \dot{q}^{i} \dot{q}^{j}
$$

The doubly covariant tensor field $g$ defines in $Q$ a Riemannian metric.

For a twice differentiable motion $t \rightarrow q^{i}=p^{i}(t)$ (or, more generally, if the functions $p^{i}$ belong to $W_{10 c}^{2,1}(\mathbb{R}, \mathbb{R})$ ), the Lagrange equations may be developed in the form

$$
g_{i j} \ddot{p}^{j}+\left(g_{i j, k}-\frac{1}{2} g_{j k, i}\right) \dot{p}^{j} \dot{p}^{k}=f_{i} .
$$

The functions $f_{i} \in L_{\text {loc }}^{1}(\mathbb{R}, \mathbb{R})$ are the covariant components, relative to the local coordinates in use, of the efforts acting on the system (possibly defined in an indirect way, through the phenomenological laws governing the physical environment).

Similarly to what has been done in Sec. 4 for a single particle, we are going to show that these equations are implied by a more general formulation, valid even in the absence of the second derivatives $\ddot{p}$.

To the local coordinates invoked above in $Q$ correspond local coordinates in the product manifold $\mathbb{R} \times Q$, denoted by $\left(q^{0}, q^{1}, \ldots, q^{n}\right)$, with $q^{0}$ ranging through $\mathbb{R}$. Greek indices will take their values in $\{0,1, \ldots, n\}$ and Latin ones in $\{1, \ldots, n\}$. Any motion $t \rightarrow p(t) \in Q$ may equivalently be 
represented by the mapping $t \rightarrow \pi(t)=(t, p(t)) \in \mathbb{R} \times Q$. Let us equip the manifold $\mathbb{R} \times Q$ with a Riemannian metric, by adjoining to the above matrix $g_{i j}$ a row and a column as follows: $g_{\alpha 0}=g_{0 \alpha}=0$ if $\alpha>0$ and $g_{00}=1$.

Using Lagrange equations amounts to reduce the dynamics of the system to that of a particle with unit mass, moving in the Riemannian manifold $Q$. So, similarly to what has been done in Sec.4, we shall associate with every continuous motion $p: \mathbb{R} \rightarrow Q$ its presence measure $\theta \in \mathcal{D}^{\circ}\left(\mathbb{R} \times Q, \mathbb{R}_{+}\right)$. This equals the functional assigning to every $\varphi \in \mathcal{D}^{0}(\mathbb{R} \times Q, \mathbb{R})$ the real number $\int_{\mathbb{R}} \varphi(\pi(t)) d t$. Then, $\theta$-almost every point of $\mathbb{R} \times \mathbb{Q}$ has the form $\xi=\pi(t), t \in \mathbb{R}$. Provided that the functions $p^{i}$, or equivalently the functions $\pi^{\alpha}$, belong to $W_{10 c}^{1}{ }^{1}(\mathbb{R}, \mathbb{R})$, one may assign to such a $\xi$ the velocity components $u^{\alpha}(\xi)=\dot{\pi}^{\alpha}(t)$ (observe that $u^{0} \equiv 1$ ), defining the element $\mathbf{u}(\xi)$ of the tangent space $(\mathbb{R} \times Q)_{\xi}$. If , more specially, the functions $p^{\prime}$, or equivalently the functions $\pi^{\alpha}$, belong to $W_{\text {loc }}^{1,2}$, the products $u^{\alpha} u^{\beta}$ belong to $L_{\text {loc }}^{l}(\mathbb{R} \times Q, \theta ; \mathbb{R})$, ensuring that the real measures $C^{\alpha \beta}=u^{\alpha} u^{\beta} \theta$ make sense. These are the components of the tensor measure $C=\mathbf{u} \otimes \mathbf{u} \theta$, an element of $\mathcal{D}^{\prime 0}\left(\mathbb{R} \times Q,(\mathbb{R} \times Q)^{\prime} \otimes_{s}(\mathbb{R} \times Q)^{\prime}\right)$ called as before the kinetic tensor measure. If (7.6) is used to express the components (equ $)_{\beta}$, elements of $\mathcal{D}^{\prime}(\mathbb{R} \times Q, \mathbb{R})$, one finds, if $\beta=i>0$,

$$
(\text { equC })_{i}=g_{i j}{ }^{j k}{ }_{, k}+\left(g_{i j, k}-\frac{1}{2} g_{j k, i}\right) C^{j k}
$$

For $\beta=0$, there simply comes out

$$
(\text { equC })_{0}=\left(u^{\gamma} \theta\right)_{\gamma} \text {. }
$$

This is the divergence of the vector measure $\mathbf{v} \theta$ (a concept independent of the Riemannian metric). Similarly to the Galilean case, $\mathbf{u} \theta$ may be called the mass current and its divergence the mass input. Here, the same integration by part as in Sec. 4 yields that this divergence vanishes

$$
\operatorname{div}(\mathbf{u} \theta)=(\text { equ })_{0}=0
$$

The reason of this fact is that the window we are using equals the whole of $\mathbb{R} \times \mathbf{Q}$.

If $p^{i}$ happens to belong to $W_{10 c}^{2,1}(\mathbb{R}, \mathbb{R})$, the same integration by parts as in (4.5) yields that $C^{j k}{ }_{, k}$ equals the real measure $\varphi \rightarrow \int_{\mathbb{R}} \ddot{p}^{j}(t) \varphi(\pi(t)) d t$. So, 
(equC) is found equal to the real measure $\varphi \rightarrow \int_{\mathbb{R}} L_{i}(t) \varphi\left(\pi(t) d t\right.$, where $L_{i}$ denotes the left-hand member of (8.2). This shows that, in such a smooth case, the Lagrange equations are equivalent to the writing

$$
\text { equC }=F,
$$

where the element $F$ of $\mathcal{D}^{\prime}\left(\mathbb{R} \times Q, \mathbb{R} \times Q^{\prime *}\right)$ is defined as follows: the components $F_{i}, i>0$, equal the measures defined on $\mathbb{R} \times Q$ as the functionals $\varphi \rightarrow \int_{R} f_{i}(t) \varphi(\pi(t)) d t$ and, by convention, $F_{0}=0$.

We therefore propose to accept (8.4) as governing the dynamics of the considered system in less smooth situations too.

Let the covector distribution $F$ be a measure, nonnecessarily admitting as above a density relative to $\theta$. There comes out, as in $\operatorname{Sec} .4$, that a motion satisfies (8.4) if and only if $t \rightarrow p^{\prime}(t)$ are functions with derivatives $\dot{p}_{i} \in \operatorname{lbv}(\mathbb{R}, \mathbb{R})$, verifying the measure differential equations corresponding to (8.2), namely

$$
g_{i j} d \dot{p}^{j}+\left(g_{i j, k}-\frac{1}{2} g_{j k, j}\right) \dot{p}^{j} \dot{p}^{k} d t=\pi^{-1}\left(F_{i}\right) .
$$

This includes in particular the traditional treatment of shocks, by means of the concept of percussion. The connection of this extension of Analytical Dynamics with the principle of Hamilton will be made clear in Sec. 11.

In particular, the confinement of the system by a boundary, as introduced in Sec.6, may be analyzed in the present setting. Let the permitted region of $\mathbb{R} \times Q$ be defined by the inequality $D(\xi) \leqslant 0$, with $D \in C^{2}(\mathbb{R} \times Q, \mathbb{R})$. At every point of the limiting hypersurface $S=\{\xi \in \mathbb{R} \times Q$ : $D(\xi)=0\}$ it is assumed that at least one of the partial derivatives $b_{i}$, for $i>0$, do not vanish. Such an inequality naturally arises when one expresses, in the framework of Analytical Dynamics, the mutual impenetrability of two parts of the investigated system in physical space, or also the confinement of one of these parts by some external obstacle. Let us split the distribution $F$, in (8.4), into the sum of a term $E$, representing regular efforts, and of a term $R$ corresponding to possible contact or impact 
effects. Depending on the circumstances which prevail in physical space, the law governing $R$ may happen to be similar to what has been formulated in Definition 6.1, conferring to the hypersurface $S$ of $\mathbb{R} \times Q$ the status of an ideal boundary.

With a view to precisely transpose Definition 6.1, let us denote by $\mathcal{A}_{0}$ the set of the test fields $n \in \mathcal{D}^{\prime}\left(\mathbb{R} \times Q,(\mathbb{R} \times Q)^{\prime}\right)$ satisfying the two following conditions:

$$
\begin{array}{ll}
\forall \xi \in \mathbb{R} \times Q & : \quad \eta^{0}(\xi)=0, \\
\forall \xi \in S: & \eta(\xi), \nabla b(\xi) \geqslant 0
\end{array}
$$

(the dot refers to the pairing of the tangent space and the cotangent space at every point of $\mathbb{R} \times \mathbf{Q}$ ).

DEFINITION 8.1. The considered unilateral constraint will be saldideal If the reasibility or an element $\mathrm{R}$ or $\mathcal{D}^{\prime}\left(\mathbb{R} \times \mathbf{Q}, \mathbb{R} \times Q^{\prime *}\right)$ ror representing the associated contact or impact effects is characterized by

$$
\begin{aligned}
& R_{0}=0, \\
\forall \eta \in \mathcal{A}_{0}: & \langle R, \eta\rangle \leqslant 0 .
\end{aligned}
$$

In common applications, this is found equivalent to the fact that, in physical space, the possible contact or impact of the concerned bodies displays no friction nor adhesion.

Using this property in order to eliminate $R$, one obtains:

PROPOSITION 8.2. The motion with kinetic tensor measure $\mathrm{C}$ is dynamically feasible in the presence of the above unilateral constraint if and only if

$$
\forall n \in \mathcal{A}_{0}: \quad\langle e q u C-E, \eta\rangle \leqslant 0
$$

In the line of Prop. 6.2, condition (8.10) is found to imply that equ $C-E$ is a measure. Therefore, as soon it is ascertained that also $E$ is a measure, the functions $t \rightarrow \dot{p}^{\prime}(t)$ corresponding to such a motion belong to $\operatorname{lbv}(\mathbb{R}, \mathbb{R})$.

Incidentally, in view of the definition of "equ", $(8.10)$ is equivalent to 


$$
\forall n \in \mathcal{A}_{0}:\langle E, \eta\rangle+\langle C, \text { def } \eta\rangle \geqslant 0
$$

The special case where the function $\mathrm{b}$ is a constant with regard to $t$ deserves notice. Due to the scleronomy assumption, this happens, in particular, if the inequality $b \leqslant 0$ expresses the mutual impenetrability of two parts of the system in physical space or also if it expresses the confinement of some of these parts by a rixed external obstacle. Then, one may introduce, instead of $\boldsymbol{A}_{0}$, the set $\mathcal{A}$ defined by imposing on $\eta$ the condition (8.7) alone. In view of (8.3) and because the component of rank zero of $E$ has been assumed to vanish, there comes out that $(8.10)$, in this case, is equivalent to

$$
\forall \eta \in \mathcal{A}: \quad\langle\text { equ } C-E, \eta\rangle \leqslant 0 .
$$

Let us stress that the preceding provides only an expression of Dynamics. In the case of impact, condition ( 8.10 ) has to be complemented with some phenomenological shock law, e.g. the assumptions that bounces are elastic or that they are soft.

\section{The transport technique.}

Let $\mathcal{M}$ be a $\mathcal{C}^{d}$-manifold, $d \geqslant 2$, with dimension $n$. A vector field $n \in \mathcal{D}^{\sigma}\left(\mathcal{M}, \mathcal{M}{ }^{\prime}\right), 1 \leqslant \sigma \leqslant d-1$, may be seen as the velocity field of a continuous medium $\Lambda$, in motion throughout $\mathcal{M}$. This means that, for every element $\lambda$ of $\Lambda$, called a particle, the placement mapping $\tau \rightarrow p(\tau, \lambda) \in \mathcal{M}$ is a solution to $d x / d \tau=n(x)$, a differential equation in $\mathcal{M}$. In other words

$$
\frac{\partial p(\tau, \lambda)}{\partial \tau}=n(p(\tau, \lambda))
$$

holds for every $\tau \in \mathbb{R}$ and every $\lambda \in \Lambda$. Both members are elements of the tangent space to $\mathcal{M}$ at point $p(\tau, \lambda)$. Here, time is denoted by $\tau$, in order to prevent, in further applications, any confusion with the time variable $t$ of Dynamics.

Through the use of local coordinates in $\mathcal{M},(9.1)$ is reduced to a differential equation in $\mathbb{R}^{n}$. Standard facts, concerning the dependence of 
solutions with regard to initial conditions, imply the following. Denote by $p_{\tau}$ the mapping $\lambda \rightarrow p(\tau, \lambda)$ of $\Lambda$ onto $\mathcal{M}$. One finds that, for every $\tau$ and $\tau^{\prime}$ in $\mathbb{R}$, the mapping $p_{\tau}, \circ p_{\tau}^{-1}$ is a $C^{\alpha}$-diffeomorphism of $\mathcal{M}$ (leaving invariant every point of $\mathcal{M} \backslash$ supp $\eta$ ).

An equivalent statement is that the continuous medium $\wedge$ may be equipped with the structure or a $C^{\sigma}$ - differential manirold, in such a way that every mapoing $p_{\tau}, \tau \in \mathbb{R}$, is a $C^{\sigma}$ - diffeomorphism of $\Lambda$ onto $\mathcal{M}$.

Clearly here, the medium $\Lambda$ is considered only from the kinematical standpoint, without referring to any material realization.

More generally, $\eta$ may also depend on the time $\tau$, this variable ranging, instead of the whole of $\mathbb{R}$, in some open real interval I (containing 0 ). The smoothness assumption made in this case is that the vector field $\lambda \rightarrow \eta(\tau, \lambda)$ has its support contained in a $\tau$-constant compact subset of $\mathcal{M}$ and that the vector field $(\tau, \lambda) \rightarrow(1, n(\tau, \lambda))$ of the product manifold $1 \times \mathcal{M}$ is $c^{\sigma}$.

DEFINITION 9.1. Such manifold as $\wedge$ above, whose motion over $\mathcal{M}$ is derined through some velocity rield $n \in \mathcal{D}^{\sigma}(\mathcal{M}, \mathcal{M})$, possibly depending on $\tau$. is calleda carrier or order 0 .

Every object of the $C^{\sigma}$-differential geometry of $\wedge$ possesses under each diffeomorphism $p_{\tau}, \tau \in I$, an image or push-forward, which is an object of the same nature in the $C^{\sigma}$-differential geometry of $\mathcal{M}$ (recall that $\sigma \leqslant d-1)$. A $\tau$-dependent object in $\mathcal{M}$, equal to the image under $p_{\tau}$ of some $\tau$-constant object in $\Lambda$, is called a moving object convected by the carrier $\Lambda$. This agrees with the meaning that the word "convected" has in Continuum Mechanics.

Consider, in particular, a specified particle $\lambda \in \Lambda$ and a specified element $x$ of the tangent space $\Lambda_{\lambda}^{\prime}$. The $C^{\sigma}$ mapping $p_{\tau}: \Lambda \rightarrow \mathcal{M}$ induces a linear mapping of $\Lambda_{\lambda}^{\prime}$ to $\mathcal{M}_{\mathrm{p}(\mathrm{\tau}, \lambda)}^{\prime}$, said tangent to $p_{\tau}$ at point $\lambda$; we shall denote this linear mapping by $p^{\prime}(\tau, \lambda)$ (or $\partial p(\tau, \lambda) / \partial \lambda$, while the other partial derivative, namely $\partial p(\tau, \lambda) / \partial \tau$, will be denoted by $\dot{p}(\tau, \lambda)$; the 
latter, an element of $\mathcal{M}_{p(\tau, \lambda)}^{\prime}$, is the velocity of the moving point $\tau \rightarrow p(\tau, \lambda)$ ). Applying $p^{\prime}(\tau, \lambda)$ to $x$ yields, for every $\tau$, an element $c(\tau)$ of $\mathcal{M}_{p(\tau, \lambda)}$ which naturally will be called a moving vector in $\mathcal{M}$, attached to the moving point $\tau \rightarrow p(\tau, \lambda)$ and convected by $\wedge$. Symmetrically, a fixed element $x^{*}$ could be chosen in the cotangent space $\Lambda_{\lambda}^{*}$. Because $p_{\tau}$ is a diffeomorphism, it induces a linear mapping of this space onto $\mathcal{M}_{\mathrm{p}(r, \lambda)}^{*}$, namely the inverse transpose of $p^{\prime}(\tau, \lambda)$; the image obtained of $x^{*}$ is a moving covector, say $c^{*}(\tau)$, convected by $\Lambda$. In general, some fixed element in the tensor product of an arbitrary number of copies of $\Lambda_{\lambda}^{*}$ and $\Lambda_{\lambda}^{* *}$ yields, as image, a moving tensor of the same type in $\mathcal{M}$, attached to the moving point $\tau \rightarrow p(\tau, \lambda))$ and said convected by $\Lambda$.

PROPOSITION 9.2. With regard to local coordinates $\left(x^{i}\right)$ in $\mathcal{M}$, let a moving vector $c$, convected by $\Lambda$, have components $c^{i}(\tau)$. These are $c^{\sigma}$ functions of $\tau$, with first derivatives

$$
\frac{\partial c^{i}}{\partial \tau}=\eta_{, j}^{i} c^{j}
$$

where the partial derivatives $\eta_{, j}^{i}$ of the components or the velocity field or $\Lambda$ are calculated at point $p(\tau, \lambda)$.

symmetrically, the components $c_{k}^{*}(\tau)$ of a convected covector $c^{*}$ are $C^{\sigma}$ functions of $\tau$, with rirst derivatives

$$
\frac{\partial c_{k}^{*}}{\partial \tau}=-n_{, k}^{j} c_{j}^{*}
$$

Proof. The element $x$ of $\Lambda_{\lambda}$ may be identified with the derivative at $r=0$ of a differentiable function $r \rightarrow \bar{\lambda}(r) \in \Lambda$, defined on a neighbourhood of 0 in $\mathbb{R}$, and such that $\hat{\lambda}(0)=\lambda$. Put $\hat{x}(\tau, r)=p(\tau, \hat{\lambda}(r))$. In view of the def inition of the image of $x$, the component $c^{\prime}(\tau)$ of this image equals the partial derivative $\partial \bar{x}^{-1} / \partial r$, calculated for $r=0$. Now $\tau \rightarrow \tilde{x}(\tau, r)$ is, for every $r$, the motion in $\mathcal{M}$ of the particle $\bar{\lambda}(r)$ of $\Lambda$, so this function makes a solution to the differential equation (9.1). Then (9.2) is nothing but the classical formula governing the dependence of such a solution with regard to the parameter $r$ (this formula is simply established by putting the differential equation into 
integral form, and by deriving the integral relatively to $r$ ). The derivation relative to $\tau$ may actually be performed $\sigma$ times, because the functions $\eta^{j}$ are $C^{\sigma}$, by assumption.

Furthermore, if $\mathrm{C}$ and $\mathrm{c}^{*}$ respectively are a vector and a covector, convected by $\Lambda$, attached to the same particle $\lambda$, then $c \cdot c^{*}=\mathcal{K} \cdot \mathcal{K}^{*}$ is a constant with regard to $\tau$. Hence, taking derivatives relatively to $\tau$, one obtains $\dot{c}^{k} c_{k}^{*}+c^{k} \dot{c}_{k}^{*}=0$ for every such pair. By identification this implies (9.3), provided that the existence of $\dot{c}_{k}^{*}$ is secured. The latter existence may be established by successively taking as $x$ the $n$ elements of a base in $\Lambda_{\lambda}^{\prime}$. This yields $n$ convected vectors denoted by $c_{(1)}, \ldots, c_{(n)}$. The components $c_{k}^{*}$ verify $n$ linear equations $c_{(i)}^{k} c_{k}^{*}=$ constant. The matrix $c_{(i)}^{k}$ is nonsingular and its elements are differentiable functions of $\tau$. Hence the same is true for its inverse, so the proof is complete.

REMARK 9.3. The above holds even if $\eta$ depends on $\tau$, under the smoothness assumptions made before Definition 9.1. More generally, formulas similar to (9.2) and (9.3) express the $\tau$-derivatives of the components of a convected tensor of any sort, attached to the moving point $\tau \rightarrow p(\tau, \lambda)$. For instance, if $C_{j}^{i}(\tau)$ are the components of a convected tensor of second order and mixed type, one has

$$
\dot{C}_{j}^{i}=n_{, k}^{i} C_{j}^{k}-n^{k},{ }_{, j}^{i}
$$

REMARK 9.4. The derivatives $d c^{i} / d \tau$ in (9.2) do not make the components of a vector which would be associated with the moving vector $\tau \rightarrow c(\tau)$ in a way independent of the coordinates in use. In fact, since $c(\tau)$ is an element of $\mathcal{M}_{\mathrm{p}(\tau, \lambda)}^{\prime}$, a $\tau$-dependent space, it can possess a $\tau$-derivative only with reference to some connection in the manifold $\mathcal{M}$. Let us observe however that, in usual Continuum Mechanics, $\mathcal{M}$ happens to be the familiar Euclidean three-dimensional space. In this special case, (9.2) expresses the components of the same vector whichever is the Cartesian (nonnecessarily orthonormal) coordinate system in use. This vector simply is the derivative of $\tau \rightarrow c(\tau)$ with regard to the standard connection of the familiar Euclidean 
space.

What precedes concerns local objects, associated with a fixed $\lambda \in \Lambda$. Let us now consider $\tau$-constant fields of the manifold $\Lambda$.

For instance, let a vector field $\lambda \rightarrow \zeta(\lambda) \in \Lambda_{\lambda}$ be given, first without adding any smoothness assumption. The push-forward of this field under the diffeomorphism $p_{\tau}: \wedge \rightarrow \mathcal{M}$ is naturally def ined as the vector field $z(\tau,$.$) ,$ assigning to every $x$ in $\mathcal{M}$ the element $p^{\prime}(\tau, \lambda)(\zeta(\lambda))$ of $\mathcal{M}_{x}^{*}$, with $\lambda=p_{\tau}^{-1}(x)$. This $\tau$-dependent vector field on $\mathcal{M}$ will be said convected by the carrier $\Lambda$. Formula (9.2) yields the derivative of the real function $\tau \rightarrow z^{i}\left(\tau, p_{\tau}(\lambda)\right)$, for $\lambda$ fixed in $\Lambda$. The existence of the derivative of $\tau \rightarrow z^{i}(\tau, x)$, for $x$ fixed in $\mathcal{M}$, is conditioned by additional smoothness assumptions. In fact, if the functions $z^{\prime}$ are $C^{\prime}$, the chain rule yields

$$
\frac{\partial z^{i}(\tau, x)}{\partial \tau}=\eta_{, j}^{i} z^{j}-\eta^{k} z_{, k}^{i},
$$

where all terms in the right-hand side are evaluated at point $(\tau, x)$. Since $z(\tau, x)$, for fixed $x$, belongs whichever is $\tau$ to the same linear space $\mathcal{M}_{x}^{\prime}$, this expresses the components of $\partial z(\tau, x) / \partial \tau$, a vector independent of the coordinates used. It is well known as the Lie bracket of the vector fields $z$ and $n$.

As other examples of fields in $\mathcal{M}$ convected by $\Lambda$, one may consider the images under $p_{\tau}$ of a covector field and of a scalar field, both def ined in $\triangle$ independently of $\tau$. In particular, it turns out that, if the $\tau$-dependent element $s_{\tau}$ of $C^{\prime}(\mathcal{M}, \mathbb{R})$ is convected, its gradient $\nabla s_{\tau} \in C^{0}\left(\mathcal{M}, \mathcal{M}^{\prime *}\right)$ is a convected covector field.

Strictly, the roles of $\mathcal{M}$ and $\triangle$ cannot be exchanged, since the differentiabllity order of $\Lambda$, by construction, is smaller than that of $\mathcal{M}$. However, one may symmetrically start with a $\tau$-constant vector field $\left.z \in C^{\prime}(\mathcal{M}, \mathcal{M})^{\prime}\right)$ and consider its pull-back under $p_{\tau}$, a $\tau$-dependent vector field $\lambda \rightarrow \zeta(\tau, \lambda) \in \Lambda_{\lambda}^{\prime}$ in $\Lambda$. Through standard arguments of Differential Calculus, the differentiability properties found in the preceding imply that, for 
every fixed $\lambda$, the derivative $(\partial \zeta(\tau, \lambda) / \partial \tau)_{\tau=0}$ exists. As a function of $\lambda$, it makes an element of $C^{0}\left(\Lambda, \Lambda^{\prime}\right)$. The $C^{0}$ vector field in $\mathcal{M}$ obtained as its push-forward under $p_{0}$ is nothing but the Lie derivative $L_{\eta} z$.

Differential Geometers are used to define the Lie derivation associated with a vector field $n$ through the consideration of the flow, or evolution operator, generated by $\eta$, without explicitely introducing the moving manifold $\Lambda$. The present equivalent construction should look more familiar to Continuum Mechanists and, in our views, makes the further proofs easier.

As another example, let us consider, instead of a vector field, the tensor field $\mathrm{g}$ used in $\operatorname{Sec} .7$ to define in $\mathcal{M}$ a Riemannian metric. Its pull-back under $p_{\tau}$ is a $\tau$-dependent tensor field $\lambda \rightarrow \gamma_{\tau}(\lambda) \in \Lambda^{* *} \otimes_{S} \Lambda^{* *}$. This corresponds to the $\tau$-dependent Riemannian metric induced on $\wedge$ by each of its placements in $\mathcal{M}$. There comes out that the $\tau$-derivative $\dot{\gamma}_{\tau}$ exists. Its push-forward under $p_{\tau}$ is the Lie derivative $L_{\eta} g$. Thereby is explained the connection of the latter with the time-rate of deformation of the continuous medium.

Let us terminate this section by observing that the pull-back and the push-forward, under a diffeomorphism of adequate order, may also be defined for tensor distributions. Consider, for instance, an element $\Theta$ of $\mathcal{D}^{\prime 0}\left(\Lambda, \Lambda^{\prime} \otimes \Lambda^{\prime}\right)$, i.e. a doubly contravariant tensor measure on $\Lambda$. By associating with every $x \in \mathcal{D}^{0}\left(\Lambda, \Lambda^{\prime *} \otimes \Lambda^{\prime *}\right)$ its push-forward, say $p_{\tau}(x)$, under the diffeomorphism $p_{\tau}: \Lambda \rightarrow \mathcal{M}$, one defines a one-to-one linear mapping of this space to $\mathcal{D}^{0}\left(\mathcal{M}, \mathcal{M}^{* *} \otimes \mathcal{M}^{* *}\right)$, bi-continuous in the pseudo-topologies we have referred to in Sec.7. Then, the push-forward $T=p_{\tau}(\Theta)$ is defined as the element of $\mathcal{D}^{0}(\mathcal{M}, \mathcal{M} \otimes \mathcal{M})$ such that

$$
\forall x \in \mathcal{D}^{0}\left(\Lambda, \Lambda^{* *} \otimes \Lambda^{* *}\right):\left\langle T, p_{\mathrm{\tau}}(x)\right\rangle=\langle\Theta, x\rangle .
$$

If $\Theta$ is $\tau$-constant, then $T$ is called a doubly contravariant tensor measure moving in $\mathcal{M}$, convected by the carrier $\Lambda$. 


\section{A variational formula.}

As in Sec.7, let us equip the manifold $\mathcal{M}$ with a Riemannian metric by fixing an element $g$ of $\mathcal{C}^{r}\left(\mathcal{M}, \mathcal{M}^{\prime *} \otimes_{s} \mathcal{M}^{* *}\right)$. Let $T \in \mathcal{D}^{0}\left(\mathcal{M}, \mathcal{M}^{\prime} \otimes_{s} \mathcal{M}^{\prime}\right)$, i.e. $T$ is a doubly contravariant symmetric tensor measure on $\mathcal{M}$. At the first stage, this tensor measure will, for simplicity, be assumed to have compact support. Then $\int g: d T=\int g_{\alpha \beta} d T^{\alpha \beta}$ is a meaningful real number that we shall call the trace integral of $T$, relative to the Riemannian metric of $\mathcal{M}$. We are going to study the $\tau$-derivative of this real functional when the tensor measure $T$ is convected by a carrier.

PROPOSITION 10.1. Let $\mathrm{T}_{\tau}$ denote a $\tau$ - depending doubly contravariant symmetric tensor measure, with compact support in $\mathcal{M}$, convected by a carrier with velocity rield $n \in \mathcal{D}^{\sigma}\left(\mathcal{M}, \mathcal{M}^{\prime}\right)$. Then

$$
\frac{d}{d \tau} \int g: d T_{\tau}=-2\left\langle e q u T_{\tau}, \eta\right\rangle \text {. }
$$

Proof. By assumption, $T_{\tau}$ equals the push-forward under $p_{\tau}$ of some $\tau$-constant $\theta \in \mathcal{D}^{\prime}\left(\Lambda, \Lambda^{\prime} \otimes_{s} \Lambda^{\prime}\right)$. Denoting, as before, by $\gamma^{\tau}$ the pull-back of $g$ under $p_{\tau}$, one has

$$
\int_{\mathcal{M}} g: d T_{\tau}=\int_{\Lambda} \gamma^{\tau}: d \theta
$$

Because $\Theta$ is a $\tau$-constant tensor measure with compact support, the $\tau$-derivative of the right-hand member equals $\int_{\Lambda} \dot{\gamma}^{\top}: d \Theta$. Justifying this derivation rests on the use of local coordinates in $\Lambda$. Since the support of $\Theta$ is compact, it is covered by the domains of a finite collection of local charts of $\Lambda$; by invoking an adequate partition of unity, one is reduced to the case of a single chart. The mean value theorem and the uniform continuity of the components of $\dot{\gamma}^{\top}$ imply that this vector field equals the $\tau$-derivative of $\gamma^{\tau}$ in the sense of the pseudo-topology of $\mathcal{D}^{0}\left(\Lambda, \Lambda^{\prime *} \otimes_{5} \Lambda^{\prime *}\right)$.

Now, pushing forward by $p_{\tau}$, one obtains

$$
\int_{\wedge} \dot{\gamma}^{\top}: d \Theta=2 \int_{\mathcal{M}} \operatorname{def} n: d T=2\langle T, \operatorname{def} n\rangle=-2\langle\text { equ } T, n\rangle \text {. }
$$

REMARK 10.2. The velocity field $\eta$ above may depend on $\tau$, under the same smoothness assumptions as in in Sec.9, involving that, for every $\tau$ in the 
concerned interval, the support of $\eta$ is contained in a fixed compact subset $\mathrm{K}$ of $\mathcal{M}$.

The extension of Prop. 10.1 to a measure $T$ with non compact support is only a matter of definition. Since the integral on the left-hand side of (10.1) is no more sure to make sense, a real function $\alpha \in \mathcal{D}^{d}(\mathcal{M}, \mathbb{R})$ with value 1 throughout $K$ is to be chosen. Applying (10.1) to the tensor measure $\alpha T$ yields a local version of Prop. 10.1; only the choice of $\alpha$ has to be adapted to that of $\eta$.

\section{Hamilton's principle.}

Various statements, concerning systems of finite freedom or continuous media, have been placed under this name. Also the status of the formulated assertions varies, depending on authors, from that of an occasional corollary to that of the very basis of Dynamics.

The general idea is to characterize the dynamically feasible motions by a property of the variations that a certain real functional undergoes, when the investigated motion is submitted to a certain class of alterations. In the line of what precedes, we are going to perform such alterations through the transport by carriers. The connection of our "equ" formulation of Dynamics with statements of Hamilton's style will thus be made clear.

For brevity, let us restrict ourselves to the setting of Sec.8. A motion of the investigated system is described as a $W_{\text {lac }}^{1,2}$ mapping $\pi: \mathbb{R} \rightarrow \mathbb{R} \times Q$. A time interval $\left[t_{0}, t_{1}\right]$ is specified and, in the manifold $\mathbb{R} \times Q$, we shall use as window the open band

$$
W=\left\{\left(q^{0}, q\right) \in \mathbb{R} \times Q: t_{0}<q^{0}<t_{1}\right\} .
$$

The components $C^{\alpha \beta}$ of the corresponding kinetic tensor measure consist of the functionals

$$
\varphi \rightarrow \int_{\left[t_{0}, t_{1}\right]} \dot{\pi}^{\alpha}(t) \dot{\pi}^{\beta}(t) \varphi(\pi(t)) d t .
$$

Consequently, the trace integral of $C$ equals 


$$
\int g: d C=\int_{\left[t_{0}, t_{1}\right]} g_{\alpha \beta}(\pi(t)) \dot{\pi}^{\alpha}(t) \dot{\pi}^{\beta}(t) d t .
$$

In this context, Proposition 10.1 yields a characterization of the dynamically feasible motions in the following variational form.

PROPOSITION 11.1. The distribution equality (8.4), governing the dynamics of the considered system, holds in the open subset $W$ of $\mathbb{R} \times Q$ if and only if, for every carrier with velocity field $n \in \mathcal{D}^{1}\left(\mathbb{W},(\mathbb{R} \times Q)^{\prime}\right)$, one has

$$
\frac{1}{2}\left(\frac{d}{d \tau}\right) \int_{\tau=0} g: d C_{\tau}=-\langle F, n\rangle
$$

Here $c_{\tau}$ denotes the doubly contravariant tensor measure, convected by the carrier, which reduces to $C$ for $\tau=0$.

In order to compare this statement with Hamilton's principle, let us now consider the kinetic part of the Hamiltonian action functional, namely, for every $P \in W^{1,2}\left(\left[t_{0}, t_{1}\right], Q\right)$

$$
A(P)=\int_{\left[t_{0}, t_{1}\right]} E_{c} d t=\frac{1}{2} \int_{\left[t_{0}, t_{1}\right]} g_{1 j}(p(t)) \dot{p}^{\prime}(t) \dot{p}^{j}(t) d t .
$$

Due to the definition, given in $\operatorname{Sec} .8$, of the matrix $g_{\alpha \beta}$, one has

$$
\frac{1}{2} \int g: d C=A+\frac{1}{2}\left(t_{1}-t_{0}\right) \text {. }
$$

Since the term $\frac{1}{2}\left(t_{1}-t_{0}\right)$ remains constant in the considered variations, we have to compare the transport of $C$, invoked in the above Proposition, with the application to $A$ of the traditional procedure of the Calculus of Variations. The latter consists in imbedding the investigated motion $t \rightarrow p(t) \in Q$ into a family depending on an additional real parameter, that we shall also denote by $\tau$, say $(t, \tau) \rightarrow P(t, \tau)$, such that

$$
\forall t: \quad P(t, 0)=p(t),
$$$$
\forall \tau: \quad P\left(t_{0}, \tau\right)=p\left(t_{0}\right), P\left(t_{1}, \tau\right)=p\left(t_{1}\right) .
$$

Now, a way of constructing $P$ consists in introducing a carrier in $\mathbb{R} \times Q$ and making it transport the point $\pi(t)=(t, p(t))$. Let us specially define this carrier by a velocity field $n \in \mathcal{D}(\mathbb{W},(\mathbb{R} \times Q)$ ') whose component of rank 
zero vanishes; this may be called an isochronous carrier, since its flow preserves each submanifold $q^{0}=$ constant of $W$. For every value of the real variable $\tau$ in some neighbourhood of zero, let us denote by $\psi_{\tau}$ the corresponding transport mapping. In other words $\psi_{\tau}\left(q^{0}, q\right)$ is the position at $\tau$ of the carrier particle whose placement at $\tau=0$ equals the element $\left(q^{0}, q\right)$ of $W$. Then define $P$ through

$$
\psi_{\tau}(t, p(t))=(t, P(t, \tau))
$$

Conditions (11.6) and (11.7) clearly are satisfied.

Observe that $t \rightarrow(t, p(t))=\pi(t)$ is a chain of points in $\mathbb{R} \times \mathbb{Q}$ which, if the traditional smoothness assumptions of the Calculus of Variations are made, depend in a $C^{1}$ way on the real variable $t$. Its derivative is the vector $(1, \dot{p}(t))=\dot{\pi}(t)$, an element of the tangent space $(\mathbb{R} \times Q))_{\pi(t)}^{\prime} \simeq \mathbb{R} \times \mathcal{Q}_{p(t)}^{\prime}$. Evaluating, for every $\tau$, the derivative of $t \rightarrow \Pi(t, \tau)=(t, P(t, \tau))$ yields an element of $\mathbb{R} \times Q_{P(t, \tau)}$, say $\dot{\Pi}(t, \tau)=(1, \dot{P}(t, \tau))$. In view of the definition (11.8) of $P$, this makes, for fixed $t$ and with $\tau$ playing the role of time, a moving vector convected by the carrier, such that $\dot{\Pi}(t, 0)=\dot{\pi}(t)$.

Similarly, the presence measure of $t \rightarrow \Pi(t, \tau)$, namely the $\tau$-dependent functional assigning to every $\varphi \in \mathcal{D}^{0}(\mathbb{W}, \mathbb{R})$ the real number $\int \varphi(\Pi(t, \tau)) d t$, is convected by the carrier and reduces, for $\tau=0$, to the presence measure of $t \rightarrow \pi(t)$. As a consequence, the kinetic tensor measure of $t \rightarrow \Pi(t, \tau)$ is convected by the carrier and reduces for $\tau=0$ to the kinetic tensor measure of $t \rightarrow \pi(t)$. By identifying this tensor measure with $C_{\tau}$ in Prop.11.1, one concludes that, if $t \rightarrow p(t)$ is a dynamically feasible motion of the system, one has, in view of (11.5),

$$
\left\langle\frac{d}{d \tau}\right\rangle_{\tau=0} A(P(., \tau))=-\langle F, n\rangle
$$

In order to recover from this equality the principle of Hamilton in its traditional form one has to make the special assumption that the distribution $F$ expresses efforts deriving from a (time-independent) potential energy function, say $U \in C^{1}(W, \mathbb{R})$. This means that $F=-(\nabla U) \theta$, where $\theta$ denotes the presence measure. Since, by construction, 
$\eta(P(t, \tau))=\partial P(t, \tau) / \partial \tau$, the right-hand member of (11.9) is found equal to $(d / d \tau)_{\tau=0} \int U(P(t, \tau)) d t$. So, the classical Hamilton action has zero derivative at $\tau=0$ in the course of the considered transport.

The foregoing was only meant to explain the connection of Prop. 11.1 with Hamilton's principle, without attempting to establish any precise equivalence. Actually the two statements have different scopes. Prop.11.1 properly pertains to Nonsmooth Dynamics.

In particular, this Proposition may be applied, in the line of Secs.6 and 8 , to motions submitted, with possible shocks, to the ideal unilateral constraint defined by the inequality $b(\xi) \leqslant 0$. Again, we shall split $F$ into the sum of a term $E$, representing regularly distributed efforts, and a term $R$, arising from contact or impact. Let $E$ derive from a potential energy $U(t, q)$. We are going to consider carriers whose velocity field $n$ belong to the set denoted by $\mathcal{A}_{0}$ in Sec. 8 , i.e. these carriers are isochronous and, at every boundary point of the permitted region, they flow in the outward direction. Then, similarly to Prop. 8.2 , one obtains

PROPOSITION 11.2. Let $\mathrm{P}$ be related through (11.8) to the investigated motion $\mathrm{p}$. The latter is dynamically reasible in the presence of the considered unilateral constraint if and only if the inequality

$$
\left(\frac{d}{d \tau}\right)_{\tau=0}\left[t_{0}, t_{1}\right]\left[\frac{1}{2} g: d C_{\tau}-U(t, P(t, \tau))\right] d t \geqslant 0
$$

holds for every carrier with velocity field $n \in \mathcal{A}_{0}$.

As in Sec. 8, one observes that, if the functions $b$ and $U$ are constant with regard to $t$, the set $\mathcal{A}_{0}$ may equivalently be replaced by $\mathcal{A}$, i.e. the considered carriers need not be isochronous.

of course, one may alternatively reverse the inequality in the definition (8.7) of $\mathcal{A}_{0}$, provided that inequality (11.10) is also reversed. Introducing, in that way, carriers whose flow at the boundary is directed inward might look more natural. But it would ruin the prospect of 
investigating solutions through minimization arguments. In fact, the study of the second derivative with respect to $\tau$ [15], when the support of $\eta$ is contained in the interior of the permitted region, shows that minimization, in the present context, can by no means be exchanged with maximization.

\section{References.}

[1] BEDFORD, A., Hamilton's principle in continuum mechanics, Research Notes in Math., N¹39, Pitman, Boston, London, Melbourne, 1985.

[2] BUTTAZZO, G. and D. PERCIVALE, on the approximation of the elastic bounce problem on Riemannian manifolds, J. Diff. Equations, 47 (1983), 227-245.

[3] FREMONT, M., Contact with adhesion, in: Topics in Nonsmooth Mechanics (Ed. J.J. Moreau, P.D. Panagiotopoulos and G. Strang), Birkhäuser, Basel, to appear.

[4] GIUSTI, E., Minimal surfaces and functions of bounded variation, Birkhäuser, Boston, Basel, Stuttgart, 1984.

[5] LICHNEROWICZ, A., Propagateurs et commutateurs en Relativité Générale, Institut des Hautes Etudes Scientifiques, Publications Mathématiques, $N^{\circ} 10,1961$.

[6] LICHNEROWICZ, A., Théorie des rayons en hydrodynamique et magnétohydrodynamique relativistes, Ann. Inst. Henri Poincaré, Sect.A, 7 (1967), 271-302.

[7]MARSDEN, J.E. and T.J.R. HUGHES, Mathematical Foundations of Elasticity, Prentice-Hall, Englewood Cliffs, N.J., 1983.

[8] MONTEIRO MARQUES, M. D. P., Chocs inélastiques standards: un résultat d'existence, Travaux du Séminaire d'Analyse Convexe, Univ. des Sci. et Techniques du Languedoc, vol.15, exposé $n^{\circ} 4$, Montpellier, 1985

[9] MONTEIRO MARQUES, M. D. P., Inclusões Diferenciais e Choques Inelásticos, Doctoral Dissertation, Faculty of Sciences, University of Lisbon, 
1988.

[10]MOREAU, J.J., Fluid dynamics and the calculus of horizontal variations, Int. J. Engng. Sci., 20 (1982), 389-411.

[11] MOREAU, J.J., Le transport d'une mesure vectorielle par un fluide et le theorème de Kelvin-Helmholtz, Rev. Roum. Math. Pures et Appl., 27 (1982), 375-383.

[12] MOREAU, J. J., Liaisons unilatérales sans frottement et chocs inélastiques, C.R. Acad.Sci. Paris, Sér.II, 296 (1983), 1473-1476.

[13] MOREAU, J.J., Variational properties of stationary inviscid incompressible flows with possible abrupt inhomogeneity or free surface, Int. J. Engng. Sci., 23 (1985), 461-481.

[14] MOREAU, J.J., Une formulation de la Dynamique Classique, C.R. Acad.Sci. Paris, Sér.II, 304 (1987), 191-194.

[15]MOREAU, J.J., Free boundaries and non-smooth solutions to some field equations: variational characterization through the transport method, in: (J. P. ZOLESIO, ed.) Proceedings of the IFIP WG 8.2 Working Conference on Boundary Control and Boundary Variations, SpringerVerlag, 1988.

[16]MOREAU, J.J., Bounded variation in time, in: Topics in Nonsmooth Mechanics (Ed. J.J. Moreau, P.D. Panagiotopoulos and G. Strang), Birkhäuser, Basel, 1988.

[17] MOREAU, J.J., Unilateral contact and dry friction in finite freedom dynamics, in: Non-smooth Mechanics and Applications (Ed. J.J. Moreau and P.D. Panagiotopoulos), CISM Courses and Lectures, SpringerVerlag, Wien, 1988.

[18] PANDIT, S.G. and DEO, S.G., Differential system involving impulses, Lecture Notes in Math., vol.954, Springer-Verlag, Berlin, Heidelberg, New York, 1982.

[19] PERCIVALE, D., Uniqueness in the elastic bounce problem, J. Diff. Equations, 56 (1985), 206-215.

[20] RHAM, G. de, Variétés Différentiables, Hermann, Paris, 1955. 
[21] SCHATZMAN, M., A class of nonlinear differential equations of second order in time, J. Nonlinear Analysis, Theory, Methods and Appl., 2 (1978), 355-373.

[22] VOL'PERT, A.I. and S.I. HUDJAEV, Analysis in classes of discontinuous functions and equations of Mathematical Physics, Martinus Nijhof $f$ Pub., Dordrecht, Boston, Lancaster, 1985. 
